\title{
STRICHARTZ ESTIMATES FOR WAVE EQUATIONS WITH COEFFICIENTS OF SOBOLEV REGULARITY
}

\author{
MATTHEW BLAIR
}

\begin{abstract}
Wave packet techniques provide an effective method for proving Strichartz estimates on solutions to wave equations whose coefficients are not smooth. We use such methods to show that the existing results for $C^{1,1}$ and $C^{1, \alpha}$ coefficients can be improved when the coefficients of the wave operator lie in a Sobolev space of sufficiently high order.
\end{abstract}

\section{IntRoduction}

Strichartz estimates refer to a family of dispersive estimates solutions to the wave equation. That is, estimates on functions $u:\left[-t_{0}, t_{0}\right] \times \mathbb{R}^{n} \rightarrow \mathbb{C}$ that are solutions to the following Cauchy problem

$$
\begin{aligned}
P(x, D) u(t, x) & :=\left(\rho(x) \partial_{t}^{2}-A(x, D)\right) u(t, x)=F(t, x) \in L^{1}\left(\left[t_{0}, t_{0}\right] ; H^{z}\left(\mathbb{R}^{n}\right)\right) \\
u(0, x) & =f(x) \in H^{z+1}\left(\mathbb{R}^{n}\right) \\
\partial_{t} u(0, x) & =g(x) \in H^{z}\left(\mathbb{R}^{n}\right)
\end{aligned}
$$

where $A(x, D)$ is a second order elliptic differential operator and the $P(x, D)$ is the wave operator.

In their original formulation, Strichartz estimates appear as the following

$$
\|u\|_{L_{t}^{p}\left(\left[-t_{0}, t_{0}\right] ; L_{x}^{q}\left(\mathbb{R}^{n}\right)\right)} \leq C\left(\|f\|_{H^{s+1}\left(\mathbb{R}^{n}\right)}+\|g\|_{H^{s}\left(\mathbb{R}^{n}\right)}+\|F\|_{L_{t}^{1}\left([-1,1] ; H^{s}\left(\mathbb{R}^{n}\right)\right)}\right)
$$

where $2 \leq p, q \leq \infty, n \geq 2$ and

$$
\frac{1}{p}+\frac{n}{q}=\frac{n}{2}-(s+1), \quad \frac{2}{p}+\frac{n-1}{q} \leq \frac{n-1}{2}
$$

with the exception of the endpoint $(s, p, q)=(0,2, \infty)$ in dimension $n=3$. Such estimates are well established in the case where the coefficients of the wave operator $P(x, D)$ belong to $C^{\infty}\left(\mathbb{R}^{n}\right)$. The first estimates of this type were proved by $\mathrm{R}$. Strichartz in [10], [11]. See [5], [3], and [4] and references therein for the full range of estimates.

When the assumption of $C^{\infty}$ coefficients is removed the issue becomes much more delicate. The techniques used to prove Strichartz estimates for smooth wave equations usually rely on asymptotic methods, which are inapplicable when the coefficients are of limited differentiability. The challenge is thus to find alternative approaches for proving such estimates in the low regularity setting.

One such approach is to employ the technology of "wave packets" to construct a parametrix for the wave operator that is suitable for proving estimates. This was 
used by H. Smith in [6] to show Strichartz estimates in dimensions $n=2,3$ under the assumption that the coefficients of the wave operator were at least $C^{1,1}\left(\mathbb{R}^{n}\right)$. More recently in [12], [13], and [14], D. Tataru was able to use the methods of the "FBI transform" to show that the full range of Strichartz estimates hold under the weaker assumption that the coefficients $a_{i j}$ of the wave operator $P(x, D)$ are time dependent and satisfy

$$
\partial_{t}^{k} \rho(t, x), \partial_{t}^{k} a_{i j}(t, x) \in L^{1}\left(\left[-t_{0}, t_{0}\right] ; C^{2-k}\left(\mathbb{R}_{x}^{n}\right)\right) \quad 0 \leq k \leq 2 .
$$

$C^{1,1}\left(\mathbb{R}^{n}\right)$ is the actually the lowest degree of continuity in $x$ that the coefficients of $P(x, D)$ must possess to guarantee that the Strichartz estimates hold. Indeed, it was shown by counterexamples in [7] that Strichartz estimates fail to hold for certain wave equations with coefficients in $C^{1, \alpha}\left(\mathbb{R}^{n}\right), 0 \leq \alpha<1$. This does not mean, however, that weaker estimates of this type are not available. In [14], Tataru was also able to show that when the coefficients $a_{i j}$ of the wave operator are time dependent and satisfy

$$
\partial_{t}^{k} \rho(t, x), \partial_{t}^{k} a_{i j}(t, x) \in L^{1}\left(\left[-t_{0}, t_{0}\right] ; C^{1+\alpha-k}\left(\mathbb{R}_{x}^{n}\right)\right) \quad \text { where } 0 \leq \alpha \leq 1 \text { and } k=0,1
$$

then a weaker family of Strichartz inequalities hold. Specifically, for $z \in \mathbb{C}$ we let $\langle D\rangle^{z}$ denote the Fourier multiplier operator on $\mathbb{R}^{n}$ with symbol $\langle\xi\rangle^{z}:=\left(1+|\xi|^{2}\right)^{\frac{z}{2}}$, then Tataru's results imply that

$$
\left\|\langle D\rangle^{-s-\frac{\sigma}{p}} u\right\|_{L_{t}^{p} L_{x}^{q}\left(\left[-t_{0}, t_{0}\right] \times \mathbb{R}^{n}\right)} \leq C\left(\|f\|_{H^{1}\left(\mathbb{R}^{n}\right)}+\|g\|_{L^{2}\left(\mathbb{R}^{n}\right)}+\|F\|_{L_{t}^{1} L_{x}^{2}\left(\left[-t_{0}, t_{0}\right] \times \mathbb{R}^{n}\right)}\right)
$$

where $\sigma=\frac{1-\alpha}{3+\alpha}$. Such estimates are indeed weaker as they ask for more regularization of $u$ to get an estimate on the $L_{t}^{p} L_{x}^{q}$ norm and hence we have "lost" $\frac{\sigma}{p}$ derivatives in the estimate.

The purpose of this paper is to explore opportunities for improving such results for wave operators with time independent coefficients that lie in a $L^{r}$ Sobolev space of sufficiently high order with $r \in(1, \infty)$. Specifically, we aim to prove that estimates of the form (1.5) hold with $\sigma=0$ when the coefficients lie in the space $L^{r, \kappa}\left(\mathbb{R}^{n}\right)$ (the $L^{r}$ Sobolev space of order $\kappa$ ) with $\kappa>\frac{n-1}{r}+2$. Thus by assuming some Sobolev regularity on coefficients, we can obtain Strichartz estimates with no loss of derivatives even though the continuity of the coefficients is below $C^{1,1}$. We also intend to show that when the coefficients lie in the space $L^{r, \kappa}\left(\mathbb{R}^{n}\right) \cap \operatorname{Lip}\left(\mathbb{R}^{n}\right)$ with $\kappa=\frac{n-1}{r}+1+\alpha, \alpha \in(0,1)$, then inequalities of the type (1.5) hold with $\sigma=\frac{1-\alpha}{3+\alpha}$ and $\alpha$ taken as in the definition of $\kappa$. In both cases, this gives us estimates with a loss of fewer derivatives when compared to what would be obtained by using Sobolev embedding and applying the results above.

To motivate why such inequalities should hold we first examine some of the issues lying at the heart of the previously mentioned results for operators with coefficients that satisfy (1.4). A crucial matter here is the regularity of the Hamiltonian flow induced by the wave operator. That is, the flow on the cotangent bundle of $\mathbb{R}^{n}$, $T^{*} \mathbb{R}^{n}$, determined by

$$
\frac{d x}{d t}=-H_{\xi}(t, x, \xi) \quad \frac{d \xi}{d t}=H_{x}(t, x, \xi)
$$

where $H(t, x, \xi)$ denotes the roots of the principal symbol of $P$ as a quadratic in $\tau$. Loosely speaking, the energy contained in the Cauchy data travels along these 
curves. Hence the dispersive qualities of solutions to the wave equation are largely determined by our ability to control the geometry of the flow induced by (1.6).

When $a_{i j}(t, x), \rho(t, x)$ have the properties detailed in (1.4), Gronwall's inequality shows that these curves exist and are unique for $t \in\left[-t_{0}, t_{0}\right]$. Additionally, the transformation $\chi_{t}: T^{*} \mathbb{R}^{n} \rightarrow T^{*} \mathbb{R}^{n}$ given by following the integral curves of (1.6) up to time $t$ is a diffeomorphism on $T^{*} \mathbb{R}^{n}$ that descends to a distance-preserving map on the cosphere bundle $S^{*}\left(\mathbb{R}^{n}\right)=\mathbb{R}^{n} \times \mathbb{S}^{n-1}$. By this we mean that there exists $C$ independent of $(x, \xi),(\tilde{x}, \tilde{\xi}) \in T^{*}\left(\mathbb{R}^{n}\right) \backslash\{0\}$ and $t \in\left[-t_{0}, t_{0}\right]$ such that

$$
C^{-1}|\pi(x, \xi)-\pi(\tilde{x}, \tilde{\xi})| \leq\left|\pi\left(\chi_{t}(x, \xi)\right)-\pi\left(\chi_{t}(\tilde{x}, \tilde{\xi})\right)\right| \leq C|\pi(x, \xi)-\pi(\tilde{x}, \tilde{\xi})|
$$

where $\pi(x, \xi)=\left(x, \frac{\xi}{|\xi|}\right)$ denotes projection onto the cosphere bundle. It is this control over the geometry of the flow $t \mapsto \chi_{t}$ that allows one to show these dispersive estimates.

However, when the continuity of our coefficients in the $x$-variable drops below $C^{1,1}$ this control over the geometry is not guaranteed. Indeed, uniqueness of solutions to (1.6) is not clear and we also lack the preservation of distance as above. In order to get results such as (1.5) for such operators, the situation is first reduced to finding estimates on solutions to the wave equation that are localized to dyadic frequency shells via Littlewood-Paley theory. Estimates on these dyadic pieces are then obtained by first truncating the $\widehat{a}_{i j}$ to a frequency scale which depends on the dyadic shell. The coefficients are then dilated so that their $C^{2}$ norm is uniformly bounded and the previously mentioned results can be applied to the smoothed out operator. The ideas behind this "truncation/rescaling" approach appear in the work of Bahouri and Chemin (see [1] and [2]), though Tataru was the first to use this procedure to show weighted Strichartz estimates such as (1.5) (see [13] and [14]).

We now turn our attention back to the case where the coefficients of the wave operator lie in $L^{r, \kappa}$, for some $\kappa>\frac{n-1}{r}+2$. Such coefficients have the property that

$$
\partial_{x}^{\beta}\left(a_{i j} \circ T\right)(x), \partial_{x}^{\beta}(\rho \circ T)(x) \in L_{l o c}^{1}\left(\mathbb{R}_{x_{1}} ; L^{\infty}\left(\mathbb{R}_{x^{\prime}}^{n-1}\right)\right) \quad|\beta| \leq 2,
$$

for all $T \in O(n)$ and $x=\left(x_{1}, x^{\prime}\right)$. Furthermore, for any isometry $S(x)=T(x)+b$ where $T \in O(n), b \in \mathbb{R}^{n}$

$$
\left\|\partial_{x}^{\beta}\left(a_{i j} \circ S\right)\right\|_{L_{x_{1}}^{1}\left((-1,1) ; L_{x^{\prime}}^{\infty}(-1,1)^{n-1}\right)},\left\|\partial_{x}^{\beta}(\rho \circ S)\right\|_{L_{x_{1}}^{1}\left((-1,1) ; L_{x^{\prime}}^{\infty}(-1,1)^{n-1}\right)} \leq C
$$

for some constant $C$ independent of choice of $S$.

With this in mind, reconsider the integral curves defined by (1.6). Assume that, in addition, $\left\|a_{i j}-\delta_{i j}\right\|_{L i p},\|\rho-1\|_{L i p} \leq \frac{1}{2}$ so that $A(x, D)$ is a perturbation of the Laplacian. Suppose our initial data $(x(0), \xi(0))$ is such that $\xi_{1}(0) \gg\left|\xi(0)^{\prime}\right|$ (here $\left.\xi=\left(\xi_{1}, \xi^{\prime}\right) \in \mathbb{R}^{n}\right)$, that is, $\xi(0)$ lies in a small cone about the positive $\xi_{1}$ axis. Since $H(\cdot, \xi) \in$ Lip, we can conclude that, for $t_{0}$ chosen sufficiently small (with choice based on the Lipschitz norm of the coefficients), $\xi(t)$ lies in a slightly larger cone about the $\xi_{1}$ axis for $t \in\left[-t_{0}, t_{0}\right]$. This implies that $\frac{d x_{1}}{d t}=-H_{\xi_{1}}(x(t), \xi(t))<0$ and hence the curve segment $(t, x(t), \xi(t)),-t_{0} \leq t \leq t_{0}$ admits a reparametrization in 
$x_{1}$ satisfying the differential equations

$$
\frac{d t}{d x_{1}}=-\frac{1}{H_{\xi_{1}}}, \quad \frac{d x^{\prime}}{d x_{1}}=\frac{H_{\xi^{\prime}}}{H_{\xi_{1}}}, \quad \frac{d \xi}{d x_{1}}=-\frac{H_{x}}{H_{\xi_{1}}} .
$$

But given (1.7) (with $T$ taken to be the identity) and (1.8), we can again apply Gronwall's Lemma and control the geometry of the flow determined by (1.6) when restricted to the product of $\mathbb{R}^{n}$ with a cone about the $\xi_{1}$-axis. By rotating coordinates, this line of reasoning holds equally well for any cone of comparable aperture. Therefore, we should be able to control the geometry of the flow determined by (1.6) on all of $T^{*} \mathbb{R}^{n}$. We thus expect that Strichartz estimates of the form (1.2) and (1.5) should hold, the latter with $\sigma=0$, for wave operators with these assumptions.

When the coefficients are in $L^{r, \kappa}, \kappa=\frac{n-1}{r}+1+\alpha$ we have the related property that

$$
\partial_{x}^{\beta}\left(a_{i j} \circ T\right)(x), \partial_{x}^{\beta}(\rho \circ T)(x) \in L_{l o c}^{1}\left(\mathbb{R}_{x_{1}} ; C^{1+\alpha-|\beta|}\left(\mathbb{R}_{x^{\prime}}^{n-1}\right)\right) \quad|\beta| \leq 1 .
$$

Hence we should be able to use elements of the "truncation/rescaling" approach to prove estimates of the form (1.5) with $\sigma=\frac{1-\alpha}{3+\alpha}$ and $\alpha$ as in (1.9).

A slightly different perspective on the problem will also be of use to us. Suppose again that the coefficients lie in $L^{r, \kappa}\left(\mathbb{R}^{n}\right), \kappa=\frac{n-1}{r}+1+\alpha$, and $\left\|a_{i j}-\delta_{i j}\right\|_{L i p}, \| \rho-$ $1 \|_{L i p} \leq \frac{1}{2}$ as before. By rewriting the principal operator of $-P(x, D)$ as

$$
a_{11} \partial_{1}^{2}+2 \sum_{j=2}^{n} a_{1 j} \partial_{1} \partial_{j}-\left(\rho \partial_{t}^{2}-\sum_{2 \leq i, j \leq n} a_{i j} \partial_{i} \partial_{j}\right)
$$

we can view the wave operator as an operator that is hyperbolic in $x_{1}$ when restricted to frequencies $\left\{(\tau, \xi):|\tau| \gg\left|\left(\xi_{2}, \ldots, \xi_{n}\right)\right|\right\}$ (where $(\tau, \xi)$ denotes Fourier transform variables in $t$ and $x$ ) as this means that the symbol of the operator is a quadratic in $\xi_{1}$ with 2 distinct real roots. We thus expect that the estimates (1.5) should hold for $u$ with Fourier transform supported in $\left\{(\tau, \xi):|\tau|>>\left|\left(\xi_{2}, \ldots, \xi_{n}\right)\right|\right\}$.

However, in view of (1.9), there is nothing special about the $x_{1}$-axis in this line of reasoning-we can repeat the argument above for any coordinate axis or for any direction in $\mathbb{R}^{n}$ so the estimates (1.5) should hold as long as the Fourier transform of the solution is sufficiently localized near the $\tau$-axis. By representing solutions to the wave equation as a sum of localized pieces we then expect that we should be able to establish Strichartz-type estimates with a loss of $\frac{1-\alpha}{p(3+\alpha)}$ derivatives for any solution to a wave equation with coefficients in $L^{r, \kappa}$. Similar considerations hold equally well when $\alpha>1$ and we wish to prove estimates with no loss of derivatives. This will be our approach to solving the problem.

As mentioned above, we will consider 2 types of wave operators, with $A(x, D)$ either taking the form

$$
A(x, D)=\sum_{1 \leq i, j \leq n} \partial_{i}\left(a_{i j}(x) \partial_{j}\right) \quad \text { or } \quad A(x, D)=\sum_{1 \leq i, j \leq n} a_{i j}(x) \partial_{i} \partial_{j} .
$$

The former set of operators being called divergence operators in contrast to the latter nondivergence form. For convenience, we will assume that $\rho \equiv 1$ whenever $P$ 
is in nondivergence form. We also assume the following bounds on the coefficients

$$
c^{-1}|\xi|^{2} \leq \sum_{i, j} a_{i j}(x) \xi_{i} \xi_{j} \leq c|\xi|^{2} \quad \rho(x) \geq c^{-1}>0
$$

with $c$ independent of $(x, \xi) \in \mathbb{R}^{2 n}$.

We will occasionally write our wave operator in the form

$$
P(x, D)=\rho(x) \partial_{t}^{2}-\sum_{1 \leq i, j \leq n} a_{i j}(x) \partial_{i} \partial_{j}-\sum_{j=1}^{n} b_{j}(x) \partial_{j} .
$$

The convention here will be that $b_{j}(x)=\sum_{i}\left(\partial_{i} a_{i j}\right)(x)$ when $P$ is in divergence form and $\rho \equiv 1, b_{j} \equiv 0$ when $P$ is in nondivergence form.

Our approach here will be to use wave packet techniques as pioneered in [6], which provide an effective method for representing solutions, but do not provide the desired Strichartz estimates in dimensions $n \geq 4$. For simplicity, we will consider 3 sets of instances of (1.3). However, the arguments here should be generalizable enough to show the full range of possibilities (1.3) in dimensions $n=2,3$.

Theorem 1.1. Suppose $\rho-1, a_{i j}-\delta_{i j} \in L^{r, \kappa}\left(\mathbb{R}^{n}\right)$, with $\kappa>\frac{n-1}{r}+2$. Then we have the following estimate for solutions $u$ to the Cauchy problem (1.1) with $z=0$

$$
\begin{aligned}
\left\|\langle D\rangle^{-s} u\right\|_{L_{t}^{p} L_{x}^{q}\left(\left[-t_{0}, t_{0}\right] \times \mathbb{R}^{n}\right)} & \\
\leq & \leq C\left(\|f\|_{H^{1}\left(\mathbb{R}^{n}\right)}+\|g\|_{L^{2}\left(\mathbb{R}^{n}\right)}+\|F\|_{L_{t}^{1} L_{x}^{2}\left([-1,1] \times \mathbb{R}^{n}\right)}\right)
\end{aligned}
$$

where one of the following hold

$$
\begin{array}{lll}
s=-\frac{1}{2} & p=q=\frac{2(n+1)}{n-1} & n=2,3 \\
s=0 & p=\frac{2 q}{q-6}, 6 \leq q<\infty & n=3 .
\end{array}
$$

Theorem 1.2. Suppose $\rho-1, a_{i j}-\delta_{i j} \in L^{r, \kappa}\left(\mathbb{R}^{n}\right) \cap \operatorname{Lip}\left(\mathbb{R}^{n}\right)$, with $\kappa=\frac{n-1}{r}+1+\alpha$, $0<\alpha<1$. Then we have the following estimate for solutions $u$ to the Cauchy problem (1.1) with $z=0$

$$
\begin{aligned}
& \left\|\langle D\rangle^{-s-\frac{\sigma}{p}} u\right\|_{L_{t}^{p} L_{x}^{q}\left(\left[-t_{0}, t_{0}\right] \times \mathbb{R}^{n}\right)} \\
& \quad \leq C\left(\|f\|_{H^{1}\left(\mathbb{R}^{n}\right)}+\|g\|_{L^{2}\left(\mathbb{R}^{n}\right)}+\|F\|_{L_{t}^{1} L_{x}^{2}\left([-1,1] \times \mathbb{R}^{n}\right)}\right)
\end{aligned}
$$

where $\sigma=\frac{1-\alpha}{3+\alpha}$ and $s, p, q, n$ either satisfy (1.11) or (1.12).

It is actually not difficult to show that Theorem 1.1 implies that estimates of the form (1.2) hold for coefficients of the same regularity and $s, p, q$ as in (1.11) or (1.12). However, this is not necessarily the case when $a_{i j}, \rho$ are as in Theorem 1.2, so we will restrict our attention to showing estimates on $\langle D\rangle^{-s} u,\langle D\rangle^{-s+\sigma / p} u$ as appropriate.

Unfortunately, our methods do not handle coefficients in $L^{r, \kappa}$ with $\kappa=\frac{n-1}{r}+1+\alpha$ and $\alpha=0$ or $\alpha=1$. Sobolev embedding does not provide for the estimate (1.9) with $\alpha=0$ in the former case and in the latter case it does not give us (1.7). When 
$\alpha=0$ we are thus unable to provide any results, and when $\alpha=1$, the best we can provide is Strichartz estimates with an arbitrarily small loss of derivatives.

Before proceeding, we will make a few additional assumptions about our coefficients and our solution. We will always assume that $a_{i j}, \rho \in \operatorname{Lip}\left(\mathbb{R}^{n}\right)$ satisfy

$$
\left\|a_{i j}-\delta_{i j}\right\|_{L i p},\|\rho-1\|_{L i p}<\varepsilon
$$

for some sufficiently small but fixed $\varepsilon>0$. Additionally, we will assume that $t_{0}=1$ and that $u(t, \cdot)$ is supported in the cube $\left(-\frac{3}{2}, \frac{3}{2}\right)^{n}$ for all $t \in\left(-\frac{5}{4}, \frac{5}{4}\right)$. The extra hypotheses are somewhat superfluous as the general case can be reduced to these assumptions by first showing that we can restrict our attention to solutions supported in small sets and then rescaling the problem so that (1.14) is satisfied. See [13] pp. 394-5 for a suitable approach. We will also assume that $u$ solves the homogeneous Cauchy problem with $F \equiv 0$. By Duhamel's principle it is not difficult to see that the general case reduces to such an assumption.

The paper is organized as follows, Section 2 details the main strategy of the paper, which focuses on obtaining estimates on components of the solution whose Fourier transform is highly localized. Sections 3 and 4 then uses wave packet techniques to provide the desired estimates for coefficients satisfying (1.7), eventually leading to a proof of Theorem 1.1. Section 5 then expounds on the truncation/rescaling arguments needed to prove Theorem 1.2.

We end with a few comments on the notation to be used in the following sections. Unless otherwise stated, the expression $X \lesssim Y$ means that $X \leq C Y$ for some constant $C$ depending only on the dimension $n$ and on the Lipschitz $/ L^{r, \kappa}$ bounds on the $\rho, a_{i j}$. Elements $y \in \mathbb{R}^{n}$ will often be denoted as $y=\left(y_{1}, y^{\prime}\right)$, so that $y^{\prime}$ denotes the last $n-1$ components of $y$. Also, for a function $v(t, x)$, and a subinterval $I \subset \mathbb{R}$ and a measurable set $U \subset \mathbb{R}^{n},\|v\|_{L_{t}^{r_{1}} L_{x}^{r_{2}}},\|v\|_{L_{t}^{r_{1}} L_{x}^{r_{2}}(I \times U)}$ will both abbreviate the norm on the Banach space $L^{r_{1}}\left(I ; L^{r_{2}}(U)\right)$ the former abbreviation being used when it is well understood from the context what $I$ is.

Acknowledgement. This work is a result of the author's doctoral dissertation research at the University of Washington. The author would like to thank Professor Hart Smith for his introduction to the problem and for his encouragement and guidance throughout the research.

\section{LOCALIZING THE SOLUTION}

In this section we outline the key elements in the strategy for proving Theorems 1.1 and 1.2. The main idea is to exploit the potential for $P$ to be "hyperbolic in $x_{1}$ " by reducing the problem to showing estimates on the components of $u$ where $\tau>>\left|\left(\xi_{2}, \ldots, \xi_{n}\right)\right|$.

Before we begin localizing our solution in frequency, we make a small alteration of our solution for technical reasons. Replace $u(t, x)$ by $\phi(t) u(t, x)$ where $\phi(t)$ is a smooth bump function equal to 1 on $[-1,1]$ and supported in $\left(-\frac{5}{4}, \frac{5}{4}\right)$. It now makes sense to look at $u$ as an element of a Sobolev space in $n+1$ variables. Hence $H_{t, x}^{w}$ will denote the $L^{2}$ Sobolev space of order $w$ on $\mathbb{R}^{n+1}$ in the $(t, x)$ variables and $H^{w}$ will denote the Sobolev space on $\mathbb{R}^{n}$ in only the $x$ variables. This modification 
of $u$ means that it is no longer a solution to the homogeneous problem. However, energy estimates allow us to control $[P, \phi]$ so that we have the following inequality for all $t \in \mathbb{R}$

$$
\|(P u)(t, \cdot)\|_{H^{s}} \lesssim\left(\|f\|_{H^{s+1}}+\|g\|_{H^{s}}\right) .
$$

2.1. Localization in frequency. Here we discuss our main approach to the problem, which involves localizing our solution in frequency to dyadic pieces where $P$ should be hyperbolic in $x_{1}$. Take a sequence of smooth Littlewood-Paley cutoffs $\left\{\beta_{k}\right\}_{k \geq 0}$, so that $\beta_{k}(\xi)$ is a smooth function defined on $\mathbb{R}^{n}$ with $\sum_{k} \beta_{k} \equiv 1$ and $\operatorname{supp}\left(\beta_{k}\right) \subset\left\{\xi \in \mathbb{R}^{n}: 2^{k-\frac{1}{2}} \leq|\xi| \leq 2^{k+\frac{3}{2}}\right\}$ when $k \neq 0$ and $\operatorname{supp}\left(\beta_{0}\right) \subset B_{1}(0)$. We can also take the sequence so that $\beta_{k}(\cdot)=\beta\left(2^{-k} \cdot\right)$ for $k \geq 1$ for some function $\beta \in C_{0}^{\infty}\left(\mathbb{R}^{n}\right)$.

By multiplying $\beta_{0}$ by another smooth cutoff in $\tau$, we can extend $\beta_{0}$ to be smooth cutoff supported in the unit ball in $\mathbb{R}^{n+1}$ and one in a neighborhood of the origin in $\mathbb{R}^{n+1}$, that is, there exists $\bar{\beta}_{0}(\tau, \xi)$ with the above properties such that $\bar{\beta}_{0}(0, \xi)=$ $\beta_{0}(\xi)$. Treating $\bar{\beta}_{0}(D)$ as a Fourier multiplier on $u$ in the $(t, x)$ variables, $\bar{\beta}_{0}(D)$ is a smoothing operator and hence by Sobolev embedding and energy estimates

$$
\left\|\langle D\rangle^{-s} \bar{\beta}_{0}(D) u\right\|_{L_{t}^{p} L_{x}^{q}\left([-1,1] \times\left(\mathbb{R}^{n}\right)\right)} \lesssim\|u\|_{H_{t, x}^{1}} \lesssim\left(\|f\|_{H^{1}}+\|g\|_{L^{2}}\right) .
$$

Now choose a conic cutoff $\Lambda^{+} \in C^{\infty}\left(\mathbb{R}^{n+1} \backslash\{0\}\right)$ such that $\operatorname{supp}\left(\Lambda^{+}\right) \subset\{(\tau, \xi)$ : $\left.9^{-1}|\xi| \leq \tau \leq 9|\xi|\right\}$ and is identically 1 on the slightly smaller cone $\left\{(\tau, \xi): 8^{-1}|\xi| \leq\right.$ $\tau \leq 8|\xi|\}$. Since we already have estimates on $\bar{\beta}_{0} u$ we will replace $\Lambda^{+}$by $\Lambda^{+}\left(I-\bar{\beta}_{0}\right)$ so that $\Lambda^{+}$is smooth on all of $\mathbb{R}^{n+1}$ and vanishes on in a neighborhood of the origin. Also, set $\Lambda^{-}(\tau, \xi)=\Lambda^{+}(-\tau, \xi)$. Our approach will be to get estimates on each of the following

$$
\Lambda^{+}(D) u, \Lambda^{-}(D) u, \text { and }\left(I-\Lambda^{+}(D)-\Lambda^{-}(D)-\bar{\beta}_{0}(D)\right) u .
$$

The Strichartz estimates for $\Lambda^{+} u, \Lambda^{-} u$ will require further localization. Let $\Omega(\xi) \in C^{\infty}\left(\mathbb{R}^{n}\right) \backslash\{0\}$ be a conical cutoff such that $\operatorname{supp}(\Omega) \subset\left\{\xi: \xi_{1}>200\left|\xi^{\prime}\right|\right\}$ and identically 1 on a slightly smaller cone. Set $\Gamma^{ \pm}(\tau, \xi)=\Omega(\xi) \Lambda^{ \pm}(\tau, \xi)$. By employing a family of similar cutoffs, we can represent $\Lambda^{ \pm} u$ as a finite sum of functions supported in cones in $\xi$ of comparable aperture, so it will suffice to show estimates for the localized $\Gamma^{ \pm}(D) u$. Similarly, we will show them for $\Gamma^{+} u$ as estimates for $\Gamma^{-} u$ will follow by an identical argument, so from now on we will suppress the ' + ' in $\Gamma^{+}$ and refer to it as $\Gamma$.

Set $\Gamma_{k}(D)=\beta_{k}(D) \circ \Gamma(D)$ so that $\Gamma_{k} u=\mathcal{F}^{-1}\left\{\beta_{k}(\xi) \Gamma(\tau, \xi) \widehat{u}(\tau, \xi)\right\}$. Let $\psi$ be a smooth cutoff in $x_{1}$ that is identically 1 on a neighborhood of $\left[-\frac{3}{2}, \frac{3}{2}\right]$ and supported in $(-2,2)$ so that $\psi\left(x_{1}\right) u(t, x)=u(t, x)$. We pause to mention that for any $x_{1} \in \mathbb{R}$, the partial Fourier Transform of $\psi \Gamma_{k} u$ in $t, x^{\prime}$ is supported in the set

$\operatorname{supp}\left(\widehat{\psi \Gamma_{k} u}\right)\left(\cdot, x_{1}, \cdot\right) \subset\left\{\left(\tau, \xi^{\prime}\right): \tau \geq 22\left|\xi^{\prime}\right|\right\} \cap\left\{\left(\tau, \xi^{\prime}\right): 2^{k-4-\frac{1}{2}} \leq\left|\left(\tau, \xi^{\prime}\right)\right| \leq 2^{k+4+\frac{3}{2}}\right\}$

The rest of this section will now be devoted to proving the following theorem:

Theorem 2.1. Let $\rho-1, a_{i j}-\delta_{i j} \in L^{r, \kappa} \cap$ Lip with $\kappa=\frac{n-1}{r}+1+\alpha$ where either $0<\alpha<1$ or $\alpha>1$. Suppose the following estimates hold on the $\psi \Gamma_{k} u$

$\left\|\psi \Gamma_{k} u\right\|_{L_{t}^{p} L_{x}^{q}\left([-1,1] \times \mathbb{R}^{n}\right)} \lesssim 2^{k\left(s+\frac{\sigma}{p}\right)}\left(2^{k}\left\|\Gamma_{k} u\right\|_{L_{t}^{\infty} L_{x}^{2}\left([-1,1] \times \mathbb{R}^{n}\right)}\right.$

$$
\left.+\left\|\nabla_{t, x}\left(\psi \Gamma_{k} u\right)\right\|_{L_{x_{1}}^{\infty} L_{t, x^{\prime}}^{2}\left(\mathbb{R}^{n+1}\right)}+\left\|P\left(\psi \Gamma_{k} u\right)\right\|_{L_{x_{1}}^{1} L_{t, x^{\prime}}^{2}\left(\mathbb{R}^{n+1}\right)}\right)
$$


where $\sigma$ is taken to be $\frac{1-\alpha}{3+\alpha}$ when $0<\alpha<1$ and $\sigma=0$ otherwise. Then Theorem 1.1 holds if $\alpha>1$ and Theorem 1.2 holds if $0<\alpha<1$.

2.2. Flux estimates. In order to control the $L_{x_{1}}^{\infty} L_{t, x^{\prime}}^{2}\left(\mathbb{R}^{n+1}\right)$ norm of the $\psi \Gamma_{k} u$, we need a family of estimates called "flux estimates" that exploit the fact that the Fourier transform of $\psi \Gamma_{k} u$ is supported in a region where $P$ can be viewed as a hyperbolic operator in $x_{1}$.

Proposition 2.2. Suppose $w(t, x) \in C^{2}\left(\mathbb{R}^{n+1}\right)$ satisfies $\partial_{t, x}^{\beta} w \in L_{t, x}^{2}$ for $|\beta| \leq 2$ and the coefficients of $P$ satisfy (1.14) for some $\varepsilon>0$ sufficiently small. In addition, suppose that for all $y_{1} \in \mathbb{R},\left(R_{y_{1}} w\right)\left(t, x^{\prime}\right):=w\left(t, y_{1}, x^{\prime}\right)$, the restriction of $w$ to the $x_{1}=y_{1}$ hyperplane has Fourier Transform satisfying

$$
\operatorname{supp}\left(\widehat{R_{y_{1}} w}\right) \subset\left\{\left(\tau, \xi^{\prime}\right):\left|\xi^{\prime}\right|^{2} \leq \frac{1}{3}|\tau|^{2}\right\} \backslash B_{\frac{1}{2}}(0) .
$$

Then given a subinterval $I \subset \mathbb{R}$ and any $y_{1}, r_{1} \in I$, there exists some constant $C_{I}$ depending only on $I, n$, and on the Lipschitz norm of the coefficients of $P$ such that

$$
\left\|\nabla_{t, x} w\left(\cdot, y_{1}, \cdot\right)\right\|_{L_{t, x^{\prime}}^{2}} \leq C_{I}\left(\left\|\nabla_{t, x} w\left(\cdot, r_{1}, \cdot\right)\right\|_{L_{t, x^{\prime}}^{2}}+\int_{r_{1}}^{y_{1}}\left\|P w\left(\cdot, z_{1}, \cdot\right)\right\|_{L_{t, x^{\prime}}^{2}} d z\right) .
$$

Proof. Define

$$
E\left(y_{1}\right)^{2}=\int_{x_{1}=y_{1}} \frac{1}{2} a_{11}\left(\partial_{1} w\right)^{2}+\frac{1}{2} \rho\left(\partial_{t} w\right)^{2}-\frac{1}{2} \sum_{i, j \geq 2} a_{i j}\left(\partial_{i} w\right)\left(\partial_{j} w\right) d t d x^{\prime}
$$

Differentiating this expression under the integral sign followed by an integration by parts in $t$ and $x^{\prime}$ allows us to obtain the following estimate

$$
\frac{d E^{2}}{d y_{1}} \lesssim \int_{x_{1}=y_{1}}\left|\partial_{1} w\right||P w|+\left(\left|\partial_{t} w\right|^{2}+\sum_{1}^{n}\left|\partial_{i} w\right|^{2}\right) d t d x^{\prime}
$$

We now use the support condition on $\widehat{R_{y_{1}} w}$ and Plancherel's equality to get that when $\varepsilon>0$ is chosen sufficiently small in (1.14),

$$
E\left(y_{1}\right)^{2} \geq \frac{1}{12}\left(\left\|\partial_{t} w\left(\cdot, y_{1}, \cdot\right)\right\|_{L_{t, x^{\prime}}^{2}}^{2}+\sum_{i=1}^{n}\left\|\partial_{i} w\left(\cdot, y_{1}, \cdot\right)\right\|_{L_{t, x^{\prime}}^{2}}^{2}\right)
$$

Using Cauchy-Schwartz, this allows us to conclude that

$$
E\left(y_{1}\right) \frac{d E}{d y_{1}}=\frac{1}{2} \frac{d E^{2}}{d y_{1}} \lesssim E\left(y_{1}\right)\left\|P w\left(\cdot, y_{1}, \cdot\right)\right\|_{L_{t, x^{\prime}}^{2}}+E\left(y_{1}\right)^{2}
$$

Given (2.3), we can divide through by $E\left(y_{1}\right)$ and apply Gronwall's inequality to prove the result.

2.3. Local estimates to global estimates. Before we prove the main theorem of this section, we need a few technical lemmas.

Lemma 2.3. Let $R$ denote a pseudodifferential operator in $t, x$ determined by a symbol $R(t, x, \tau, \xi) \in S_{1,0}^{0}\left(\mathbb{R}^{n+1}\right)$, and $P$ a wave operator with Lipschitz coefficients. Then

$$
[P, R]: H_{t, x}^{1} \rightarrow L_{t, x}^{2}
$$

continuously with operator norm depending on the symbol estimates and on the Lipschitz norm of the coefficients of $P$. 
Proof. As a corollary of the commutator theorem of Coifman-Meyer we have that

$$
[\rho, R],\left[a_{i j}, R\right]: H_{t, x}^{-1} \rightarrow L_{t, x}^{2} \quad \text { and } \quad[\rho, R],\left[a_{i j}, R\right]: L_{t, x}^{2} \rightarrow H_{t, x}^{1}
$$

with the desired operator norm properties (see Proposition 3.6B and the surrounding discussion in Section 3.6 of [15]). By continuity of differential operators in Sobolev spaces, the former mapping easily provides the result for nondivergence operators and the latter mapping does the same for divergence operators.

Lemma 2.4. Let $\left\{T_{k}\right\}_{k=0}^{\infty}$ be a sequence of linear operators mapping $\mathcal{S}\left(\mathbb{R}^{n}\right)$ (the Schwartz class functions on $\mathbb{R}^{n}$ ) to $\mathcal{S}^{\prime}\left(\mathbb{R}^{n}\right)$ ( tempered distributions on $\mathbb{R}^{n}$ ). Suppose for any $\theta \in \mathbb{R}, \sum_{k} e^{i k \theta} T_{k}$ is a well defined mapping from $\mathcal{S}\left(\mathbb{R}^{n}\right) \rightarrow \mathcal{S}^{\prime}\left(\mathbb{R}^{n}\right)$ that extends to a continuous map from $H^{z}\left(\mathbb{R}^{n}\right)$ to $H^{w}\left(\mathbb{R}^{n}\right)$ with operator norm uniformly bounded in $\theta$. Then there exists $C$ depending on $T_{k}$ such that

$$
\sum_{k=0}^{\infty}\left\|T_{k} h\right\|_{H^{w}\left(\mathbb{R}^{n}\right)}^{2} \leq C\|h\|_{H^{z}\left(\mathbb{R}^{n}\right)}^{2}
$$

for all $h \in H^{z}\left(\mathbb{R}^{n}\right)$.

Proof. Replacing $T_{k}$ by $\langle D\rangle^{w} T_{k}\langle D\rangle^{-z}$ if necessary it suffices to proof the lemma in the case where $z=w=0$. By Plancherel's equality we have

$$
\sum_{k=0}^{\infty}\left\|T_{k} h\right\|_{L^{2}\left(\mathbb{R}^{n}\right)}^{2} \approx \int_{0}^{2 \pi} \int_{\mathbb{R}^{n}}\left|\sum_{k=0}^{\infty} e^{i k \theta}\left(T_{k} h\right)(x)\right|^{2} d x d \theta \leq 2 \pi C\|h\|_{L^{2}\left(\mathbb{R}^{n}\right)}^{2}
$$

for some $C$ independent of $\theta$. This proves the claim.

Proof of Theorem 2.1. We first reduce matters to estimates on $\Gamma u$ by handling the term $\left(I-\Lambda^{+}-\Lambda^{-}\right) u$. The Fourier transform of this function is supported in a region where $P$ is elliptic and hence by elliptic regularity results for pseudodifferential operators with rough symbols (see Theorem 2.2B in [15], which also holds in the Sobolev space setting) we have

$$
\begin{aligned}
\left\|\left(I-\Lambda^{+}-\Lambda^{-}\right) u\right\|_{H_{t, x}^{2}} & \lesssim\left\|P\left(I-\Lambda^{+}-\Lambda^{-}\right) u\right\|_{L_{t, x}^{2}} \\
& \lesssim\left\|\left[P, I-\Lambda^{+}-\Lambda^{-}\right] u\right\|_{L_{t, x}^{2}}+\|P u\|_{L_{t, x}^{2}} \lesssim\|f\|_{H^{1}}+\|g\|_{L^{2}},
\end{aligned}
$$

the last inequality following by Lemma 2.3, energy estimates, and (2.1). This estimate along with Sobolev embedding estimate $\langle D\rangle^{-s}: H_{t, x}^{2} \hookrightarrow L_{t}^{p} L_{x}^{q}\left(\mathbb{R}^{n+1}\right)$ now implies that

$$
\left\|\langle D\rangle^{-s}\left(I-\Lambda^{+}-\Lambda^{-}\right) u\right\|_{L_{t}^{p} L_{x}^{q}\left(\mathbb{R}^{n+1}\right)} \lesssim\|f\|_{H^{1}}+\|g\|_{L^{2}} .
$$

Turning our attention to $\Gamma u$, we observe that by Littlewood-Paley theory

$$
\left\|\langle D\rangle^{-s-\frac{\sigma}{p}} \Gamma u\right\|_{L_{t}^{p} L_{x}^{q}} \approx\left\|\left(\sum_{k}\left|\langle D\rangle^{-s-\frac{\sigma}{p}} \Gamma_{k} u\right|^{2}\right)^{\frac{1}{2}}\right\|_{L_{t}^{p} L_{x}^{q}} \lesssim\left(\sum_{k} 2^{-2 k\left(s+\frac{\sigma}{p}\right)}\left\|\Gamma_{k} u\right\|_{L_{t}^{p} L_{x}^{q}}^{2}\right)^{\frac{1}{2}}
$$

so it suffices to dominate the latter sum by $\|f\|_{H^{1}}+\|g\|_{L^{2}}$.

We now use the fact that $(1-\psi) u \equiv 0$ to write $\Gamma_{k} u=\psi \Gamma_{k} u+\left[1-\psi, \Gamma_{k}\right] u$. To control the terms involving $\psi \Gamma_{k} u$, first observe that as a consequence of the flux estimates we have

$$
\begin{aligned}
\left\|\nabla_{t, x}\left(\psi \Gamma_{k} u\right)\right\|_{L_{x_{1}}^{\infty} L_{t, x^{\prime}}^{2}}^{2} & \lesssim\left\|\nabla_{t, x}\left(\psi \Gamma_{k} u\right)(\cdot,-2, \cdot)\right\|_{L_{t, x^{\prime}}^{2}}^{2}+\left\|P\left(\psi \Gamma_{k} u\right)\right\|_{L_{x_{1}}^{1} L_{t, x^{\prime}}^{2}}^{2}\left(\mathbb{R}_{t, x}^{n+1}\right) \\
& \lesssim\left\|P\left(\psi \Gamma_{k} u\right)\right\|_{L_{t, x}^{2}}^{2}
\end{aligned}
$$


with the last inequality following from the compact support of $\psi$ in $x_{1}$. Hence by $(2.2)$, the sum over $\psi \Gamma_{k} u$ is now dominated by

$$
\sum_{k} 2^{-2 k\left(s+\frac{\sigma}{p}\right)}\left\|\psi \Gamma_{k} u\right\|_{L_{t}^{p} L_{x}^{q}}^{2} \lesssim \sum_{k}\left(\left\|\Gamma_{k} u\right\|_{L_{t}^{\infty}\left([-1,1] ; H^{1}\right)}^{2}+\left\|P\left(\psi \Gamma_{k} u\right)\right\|_{L_{t, x}^{2}}^{2}\right)
$$

We first estimate $P\left(\psi \Gamma_{k} u\right)$. Begin by writing

$$
P\left(\psi \Gamma_{k} u\right)=[P, \psi] \Gamma_{k} u+\psi\left[P, \Gamma_{k}\right] u+\psi \Gamma_{k} P u .
$$

First consider the middle term. $\psi$ is a bounded function, so we wish to control the $\operatorname{sum} \sum_{k}\left\|\left[\Gamma_{k}, P\right] u\right\|_{L_{t, x}^{2}}$. Indeed, the estimate

$$
\sum_{k}\left\|\left[P, \Gamma_{k}\right] u\right\|_{L_{t, x}^{2}}^{2} \lesssim\|u\|_{H_{t, x}^{1}}^{2}
$$

follows by Lemma 2.4 once it is observed that an application of Lemma 2.3 shows $\left[P, \sum_{k} e^{i k \theta} \Gamma_{k}\right]: H_{t, x}^{1} \rightarrow L_{t, x}^{2}$ continuously with operator norm bounded by some constant independent of $\theta$. Given energy estimates and (2.1), the remaining terms in (2.4) are also easily dominated by $\|f\|_{H^{1}}^{2}+\|g\|_{L^{2}}^{2}$ when we sum their squares over $k$.

Let $\phi(t)$ be the smooth cutoff function in $t$ defined above that is identically 1 on $[-1,1]$ and zero outside $\left(-\frac{5}{4}, \frac{5}{4}\right)$. Energy estimates yield

$$
\left\|\Gamma_{k} u\right\|_{L_{t}^{\infty}\left([-1,1] ; H^{1}\right)} \lesssim\left\|P\left(\phi \Gamma_{k} u\right)\right\|_{L_{t, x}^{2}} .
$$

An estimate in the vein of $(2.4)$ and (2.5) now controls $\sum_{k}\left\|\Gamma_{k} u\right\|_{L_{t}^{\infty}\left([-1,1] ; H_{x}^{1}\right)}^{2}$.

We are now left to control the sum $\sum_{k} 2^{-2 k\left(s+\frac{\sigma}{p}\right)}\left\|\left[1-\psi, \Gamma_{k}\right] u\right\|_{L_{t}^{p} L_{x}^{q}}^{2}$. This is easily accomplished by Sobolev embedding and the fact that $\left[1-\psi, \sum e^{i k \theta} 2^{-k\left(s+\frac{\sigma}{p}\right)} \Gamma_{k}\right]$ is a pseudodifferential operator with symbol estimates in $S_{1,0}^{-s-1-\frac{\sigma}{p}}$ independent of $\theta$.

\section{PARAmetrices For the WAVE Operator}

Now that we have reduced the main theorems to showing estimates on the $\psi \Gamma_{k} u$, we wish to be able to represent such functions in a manner that is suitable for proving Strichartz inequalities. As noted in the introduction, we will use wave packet techniques as developed in [6].

Our strategy is motivated by the observations in the introduction. We first construct a pseudodifferential operator with rough symbol $\widetilde{P}$ such that $\widetilde{P}(w)=$ $\frac{1}{a_{11}} P(w)$ for functions $w$ whose partial Fourier transforms are indeed supported in a region where $\tau>>\left|\xi^{\prime}\right|$, yet is hyperbolic in the $x_{1}$ variable rather than in $t$. We then proceed to use wave packet methods to construct a parametrix for $\widetilde{P}$. Once we have a suitable representation of the solution, Section 4 will demonstrate the desired Strichartz estimates.

Throughout this section and in Section 4 we will assume that

$$
\partial_{x}^{\beta} a_{i j}(x), \partial_{x}^{\beta} \rho(x) \in L_{x_{1}}^{r} L_{x^{\prime}}^{\infty}\left(\mathbb{R}^{n}\right) \quad|\beta| \leq 2,
$$


for some $1<r<\infty$. As alluded to in the introduction, this condition holds when the coefficients of the wave operator satisfy the hypotheses of Theorem 1.1 and a smoothing procedure will ensure this holds when $a_{i j}, \rho$ satisfy the conditions of Theorem 1.2.

To construct $\widetilde{P}$, let $\Psi \in C^{\infty}\left(\mathbb{R}^{n} \backslash\{0\}\right)$ be a smooth, homogeneous of degree 0 function in $\left(\tau, \xi^{\prime}\right)$ such that $\operatorname{supp}(\Psi) \subset\left\{\left(\tau, \xi^{\prime}\right): \tau \geq 5\left|\xi^{\prime}\right|\right\}$ and is identically 1 on the slightly smaller region $\left\{\left(\tau, \xi^{\prime}\right): \tau \geq 6\left|\xi^{\prime}\right|\right\}$. Next we pick a smooth radial cutoff $\Omega$ such that $\Omega$ is identically 1 on a neighborhood of $B_{\frac{1}{2}}(0) \subset \mathbb{R}^{n}$ and zero outside $B_{\frac{3}{4}}(0)$ and replace $\Psi$ by $\Psi(1-\Omega)$ so that $\Psi$ is smooth on all of $\mathbb{R}^{n}$. Additionally,

$$
\Psi\left(\tau, \xi^{\prime}\right)=\Psi\left(\left(\tau, \xi^{\prime}\right) /\left|\left(\tau, \xi^{\prime}\right)\right|\right) \quad \text { for }\left|\left(\tau, \xi^{\prime}\right)\right| \geq \frac{3}{4}
$$

We now set $\tilde{a}_{i j}=a_{i j} / a_{11}, \tilde{\rho}=\rho / a_{11}$ and define $\widetilde{P}$ as the pseudodifferential operator with rough symbol given by

$$
\begin{aligned}
\widetilde{P}(x, \tau, \xi)=\xi_{1}^{2} & +\left(2 \sum_{j=2}^{n} \tilde{a}_{1 j}(x) \xi_{1} \xi_{j}-\left(\tilde{\rho}(x) \tau^{2}-\sum_{2 \leq i, j \leq n} \tilde{a}_{i j}(x) \xi_{i} \xi_{j}\right)\right) \Psi\left(\tau, \xi^{\prime}\right) \\
& -\left(\tau^{2}+\sum_{i=2}^{n} \xi_{i}^{2}\right)\left(1-\Psi\left(\tau, \xi^{\prime}\right)\right)+i \sum_{1}^{n} \tilde{b}_{i}(x) \xi_{i}
\end{aligned}
$$

Here either $\rho(x) \equiv 1$ and $\tilde{b}_{i} \equiv 0$ for nondivergence operators or $\tilde{b}_{i}=a_{11}^{-1} \sum_{j=1}^{n} \partial_{j} a_{i j}$ for divergence operators.

We will also need to smooth the coefficients of $\widetilde{P}$ by truncating their Fourier transform in $x$ to frequencies less than $2^{\frac{k}{2}}$. Let $\widehat{\phi}=\Omega$, define $\phi_{k}(x)=2^{\frac{n k}{2}} \phi\left(2^{\frac{k}{2}} x\right)$, and then set $\tilde{a}_{i j}^{k}=\phi_{k} * \tilde{a}_{i j}, \tilde{\rho}^{k}=\phi_{k} * \tilde{\rho}$. We need a few estimates on the $\tilde{a}_{i j}^{k}$ and $\tilde{\rho}^{k}$. We first show the existence of an $L^{r}(\mathbb{R})$ function $\varrho$ such that $\left\|\left(\tilde{a}_{i j}-\tilde{a}_{i j}^{k}\right)\left(x_{1}, \cdot\right)\right\|_{L_{x^{\prime}}^{\infty}} \lesssim$ $2^{-k} \varrho\left(x_{1}\right)$. Set

$$
\left\|D_{x}^{2} A\left(x_{1}, \cdot\right)\right\|_{L_{x^{\prime}}^{\infty}}:=\sum_{|\alpha|=2} \sum_{i, j}\left\|\partial_{x}^{\alpha} a_{i j}\left(x_{1}, \cdot\right)\right\|_{L_{x^{\prime}}^{\infty}}+\sum_{|\alpha|=2}\left\|\partial_{x}^{\alpha} \rho\left(x_{1}, \cdot\right)\right\|_{L_{x^{\prime}}^{\infty}} .
$$

and let $\varrho\left(x_{1}\right)$ denote the Hardy-Littlewood maximal function associated to the $L^{r}(\mathbb{R})$ function $x_{1} \mapsto\left\|D_{x}^{2} A\left(x_{1}, \cdot\right)\right\|_{L_{x^{\prime}}^{\infty}}$. Note that since $1<r<\infty, \varrho\left(x_{1}\right) \in L^{r}(\mathbb{R})$ as well. We next observe that for any Schwartz function $h \in \mathcal{S}(\mathbb{R})$ and $\lambda>0$

$$
\int\left\|D_{x}^{2} A\left(x_{1}-z, \cdot\right)\right\|_{L_{x^{\prime}}^{\infty}} h(\lambda z) \lambda d z \leq C_{h} \varrho\left(x_{1}\right)
$$

for some constant $C_{h}$ depending only on $h$. This is a consequence of Theorem 2 in Chapter III, Section 2 of [9].

Since $\phi$ is radial and hence $\int y_{i} \phi(y) d y=0$, a Taylor expansion of $\tilde{a}_{i j}$ with second order error yields the estimate

$$
\begin{aligned}
\left|\tilde{a}_{i j}(x)-\tilde{a}_{i j}^{k}(x)\right| & =\left|\int\left(\tilde{a}_{i j}(x)-\tilde{a}_{i j}(x-y)\right) \phi\left(2^{\frac{k}{2}} y\right) 2^{\frac{k n}{2}} d y\right| \\
& \lesssim 2^{-k} \int_{0}^{1} \int\left\|D_{x}^{2} A\left(x_{1}-(1-t) y_{1}, \cdot\right)\right\|_{L_{x^{\prime}}^{\infty}}\left(2^{\frac{k}{2}} y_{1}\right)^{2} \Phi\left(2^{\frac{k}{2}} y_{1}\right) 2^{\frac{k}{2}} d y_{1} d t
\end{aligned}
$$


with $\Phi\left(y_{1}\right)=\int\left|\phi\left(y_{1}, y^{\prime}\right)\right| d y^{\prime}$. For $0<t<1$ we make a change variables $z_{t}=$ $(1-t) y_{1}$ in the last integral and apply $(3.2)$ with $h(z)=z^{2} \Phi(z)$ to get that

$$
\left\|\tilde{a}_{i j}\left(x_{1}, \cdot\right)-\tilde{a}_{i j}^{k}\left(x_{1}, \cdot\right)\right\|_{L_{x^{\prime}}^{\infty}} \lesssim 2^{-k} \varrho\left(x_{1}\right) .
$$

Note that we also get identical results for $\rho^{k}$.

A second application of the maximal function result above allows us to conclude

$$
\left\|\partial_{x}^{\beta} \tilde{a}_{i j}^{k}\left(x_{1}, \cdot\right)\right\|_{L_{x^{\prime}}^{\infty}},\left\|\partial_{x}^{\beta} \tilde{\rho}^{k}\left(x_{1}, \cdot\right)\right\|_{L_{x^{\prime}}^{\infty}} \leq C_{\beta} 2^{\frac{k}{2}(|\beta|-2)} \varrho\left(x_{1}\right) \quad \text { for }|\beta| \geq 2 .
$$

Since $a_{i j}, \rho \in \operatorname{Lip}\left(\mathbb{R}^{n}\right)$ we also have that for $|\beta| \leq 1,\left\|\partial_{x}^{\beta} \tilde{a}_{i j}^{k}\right\|_{L^{\infty}} \leq C$ for some $C$ independent of $k$ and that

$$
\left\|\partial_{x}^{\beta} \tilde{a}_{i j}^{k}\right\|_{L^{\infty}},\left\|\partial_{x}^{\beta} \tilde{\rho}^{k}\right\|_{L^{\infty}} \leq C_{\beta} 2^{\frac{k}{2}(|\beta|-1)} \quad \text { for }|\beta| \geq 1 .
$$

Define $\widetilde{P}^{k}(x, D)$ as the pseudodifferential operator with smooth symbol obtained by replacing $\tilde{a}_{i j}, \tilde{\rho}$ by $\tilde{a}_{i j}^{k}, \tilde{\rho}^{k}$ in the definition of $\widetilde{P}$. By choosing $\varepsilon>0$ in (1.14) sufficiently small, we see that the principal symbols of $\widetilde{P}, \widetilde{P}^{k}$ are quadratics in $\xi_{1}$ with 2 real roots, that is, they are operators that are hyperbolic in $x_{1}$. We will employ wave packet techniques to construct a parametrix for $\widetilde{P}$, so to provide a more intuitive exposition we will reverse the roles of $t$ and $x_{1}$ and assume that $\tilde{P}$ is hyperbolic in $t$. So for the rest of this section we will prove results about hyperbolic operators $\widetilde{P}(t, x, \tau, \xi), \widetilde{P}^{k}(t, x, \tau, \xi)$ obtained by switching the roles of the variables $(t, \tau)$ and $\left(x_{1}, \xi_{1}\right)$.

Define $Q, Q^{k}$ as the principal symbols of $\widetilde{P}, \widetilde{P}^{k}$ respectively. Let $H_{k}^{ \pm}(t, x, \xi)$, $H^{ \pm}(t, x, \xi)$ denote the roots of the symbols of $Q^{k}, Q$ respectively as a quadratic in $\tau$. We can now write the operator $Q^{k}(t, x, D)$ as

$$
Q^{k}(t, x, D)=\left(i \partial_{t}+H_{k}^{-}(t, x, D)\right) \circ\left(i \partial_{t}+H_{k}^{+}(t, x, D)\right)+E_{k}^{+}(t, x, D)
$$

with error term $E_{k}^{+}(t, x, D)$. Therefore, to create an approximate solution operator for $\widetilde{P}_{k}$ it should suffice to construct an evolution operator which loses no derivatives when composed with $\left(i \partial_{t}+H_{k}^{+}(t, x, D)\right)$. Indeed, we will write out $E_{k}^{+}(t, x, D)$ below and argue that the error brought about by the this term and the first order terms will contribute to the loss of only one derivative.

Lastly, we observe that since the roles of $t$ and $x_{1}$ are switched, the estimates on the smoothed out coefficients of $\widetilde{P}^{k}$ take the form

$$
\begin{aligned}
\left\|\left(\tilde{a}_{i j}-\tilde{a}_{i j}^{k}\right)(t, \cdot)\right\|_{L_{x}^{\infty}},\left\|\left(\tilde{\rho}-\tilde{\rho}^{k}\right)(t, \cdot)\right\|_{L_{x}^{\infty}} & \leq C 2^{-k} \varrho(t), & & \\
\left\|\partial_{t, x}^{\beta} \tilde{a}_{i j}^{k}(t, \cdot)\right\|_{L_{x}^{\infty}},\left\|\partial_{t, x}^{\beta} \tilde{\rho}^{k}(t, \cdot)\right\|_{L_{x}^{\infty}} & \leq C_{\beta} 2^{\frac{k}{2}(|\beta|-2)} \varrho(t) & & \text { for }|\beta| \geq 2, \\
\left\|\partial_{t, x}^{\beta} \tilde{a}_{i j}^{k}\right\|_{L_{t, x}^{\infty}},\left\|\partial_{t, x}^{\beta} \tilde{\rho}^{k}\right\|_{L_{t, x}^{\infty}} & \leq C_{\beta} 2^{\frac{k}{2}(|\beta|-1)} & & \text { for }|\beta| \geq 1 .
\end{aligned}
$$

3.1. Wave Packets. Here we review the essential properties of "wave packets", which are a frame of functions on $L^{2}\left(\mathbb{R}^{n}\right)$ with properties that are well suited for analysis of wave operators. This frame will be used to construct a parametrix for $\widetilde{P}$. This section is essentially a summary of the results in Section 2 of [6]. 
To construct this frame we begin by taking a partition of unity

$$
\left|h_{0}(\xi)\right|^{2}+\sum_{k=1}^{\infty} \sum_{\omega}\left|h_{k}^{\omega}(\xi)\right|^{2} \equiv 1
$$

on $\mathbb{R}^{n}$ where $\omega$ ranges over a set of $\approx 2^{\frac{k(n-1)}{2}}$ vectors on the unit sphere, equally spaced by a distance of $\approx 2^{-\frac{k}{2}}$. These functions can be taken so that

$$
\operatorname{supp}\left(h_{k}^{\omega}\right) \subset\left\{\xi \in \mathbb{R}^{n}: 2^{k-\frac{1}{2}} \leq|\xi| \leq 2^{k+\frac{3}{2}}\right\} \cap\left\{\xi \in \mathbb{R}^{n}:|\omega-\xi /| \xi|| \leq 2^{-\frac{k}{2}}\right\}
$$

with derivatives that satisfy

$$
\left|\left\langle\omega, \partial_{\xi}\right\rangle^{j} \partial_{\xi}^{\beta} h_{k}^{\omega}(\xi)\right| \leq C_{\beta, j} 2^{-k j-\frac{k}{2}|\beta|} .
$$

Such a partition of unity is constructed in Chapter 9, Section 4.4 of [8].

For each pair $\omega, k$ let $\Xi_{k}^{\omega}$ be the rectangular lattice in $\mathbb{R}^{n}$ evenly spaced by distance $2^{-k}$ in the $\omega$ direction and spaced $2^{-\frac{k}{2}}$ is the directions orthogonal to $\omega$. Given any triplet $\gamma=\left(x_{0}, k, \omega\right)$ where $x_{0} \in \Xi_{k}^{\omega}$, set

$$
\widehat{\varphi_{\gamma}}(\xi)=(2 \pi)^{-\frac{n}{2}} 2^{-\frac{k(n+1)}{4}} e^{-i\left\langle x_{0}, \xi\right\rangle} h_{k}^{\omega}(\xi) .
$$

By using Fourier series, it can be shown that this forms a frame of functions for $L^{2}$ in the sense that if $f \in L^{2}\left(\mathbb{R}^{n}\right)$, and $c_{\gamma}=\int f(y) \overline{\varphi_{\gamma}(y)} d y$, then

$$
f(y)=\sum_{\gamma} c_{\gamma} \varphi_{\gamma}(y), \quad \int|f(y)|^{2} d y=\sum_{\gamma}\left|c_{\gamma}\right|^{2} .
$$

However, these frame elements, which we will refer to as "wave packets", are not mutually orthogonal or even linearly independent. In space, these wave packets are now localized to a region of width $2^{-k}$ in the $\omega$ direction and width $2^{-\frac{k}{2}}$ orthogonally in the sense that

$$
\left|\left(y-x_{0}\right)^{\alpha}\left\langle\omega, y-x_{0}\right\rangle^{i}\left\langle\omega, \partial_{y}\right\rangle^{j}\left\langle\omega^{\perp}, \partial_{y}\right\rangle^{\beta} \varphi_{\gamma}(y)\right| \leq C_{\alpha, \beta, i, j} 2^{\frac{k(n+1)}{4}+\frac{k}{2}(|\beta|-|\alpha|)+k(j-i)}
$$

where $\omega^{\perp}$ is an any direction orthogonal to $\omega$. We also note that this frame also allows us to represent tempered distributions in an $L^{2}$ Sobolev space with norm approximated by $\|f\|_{H^{s}\left(\mathbb{R}^{n}\right)}^{2} \approx \sum_{\gamma} 2^{2 k s}\left|c_{\gamma}\right|^{2}$.

We next observe the correspondence between matrices on $\cup_{\omega, k} \Xi_{k}^{\omega} \times \cup_{\omega, k} \Xi_{k}^{\omega}$ and operators on $L^{2}\left(\mathbb{R}^{n}\right)$. Indeed given any operator $T$ on $L^{2}\left(\mathbb{R}^{n}\right)$ we can associate a matrix to it given by

$$
b\left(\gamma, \gamma^{\prime}\right)=\int \overline{\varphi_{\gamma}(y)}\left(T \varphi_{\gamma^{\prime}}\right)(y) d y
$$

Conversely, given any matrix $\left\{b\left(\gamma, \gamma^{\prime}\right)\right\}_{\gamma, \gamma^{\prime}}$ we can formally associate an operator to it determined by

$$
T f=\sum_{\gamma, \gamma^{\prime}} b\left(\gamma, \gamma^{\prime}\right) c_{\gamma^{\prime}} \varphi_{\gamma}
$$

Below we will discuss conditions on matrices on $\cup_{\omega, k} \Xi_{k}^{\omega} \times \cup_{\omega, k} \Xi_{k}^{\omega}$ for which its associated operator is bounded on $L^{2}\left(\mathbb{R}^{n}\right)$. 
This frame of functions is well-suited for analysis on the cosphere bundle of $\mathbb{R}^{n}$, $S^{*}\left(\mathbb{R}^{n}\right)=\mathbb{R}^{n} \times \mathbb{S}^{n-1}$. We place a natural pseudodistance on the cosphere bundle given by

$$
d(x, \omega ; \tilde{x}, \tilde{\omega})=|\langle\omega, x-\tilde{x}\rangle|+|\langle\tilde{\omega}, x-\tilde{x}\rangle|+\min \left(|x-\tilde{x}|,|x-\tilde{x}|^{2}\right)+|\omega-\tilde{\omega}|^{2} .
$$

We also have the following useful estimates on $d$

$$
\begin{gathered}
d(x, \omega ; \tilde{x}, \tilde{\omega}) \approx|\langle\omega, x-\tilde{x}\rangle|+\min \left(|x-\tilde{x}|,|x-\tilde{x}|^{2}\right)+|\omega-\tilde{\omega}|^{2} \\
d(x, \omega ; \tilde{x}, \tilde{\omega}) \leq 6\left(d\left(x, \omega ; x^{\prime}, \omega^{\prime}\right)+d\left(x^{\prime}, \omega^{\prime} ; \tilde{x}, \tilde{\omega}\right)\right) .
\end{gathered}
$$

This pseudodistance can now be used to define a weight function on $\cup_{\omega, k} \Xi_{k}^{\omega}$. For $\gamma=(x, \omega, k), \gamma^{\prime}=\left(x^{\prime}, \omega^{\prime}, k^{\prime}\right) \in \cup_{\omega, k} \Xi_{k}^{\omega}$ we define

$$
\mu_{\delta}\left(\gamma, \gamma^{\prime}\right)=\left(1+\left|k-k^{\prime}\right|^{2}\right)^{-1} 2^{-\left(\delta+\frac{n}{2}\right)\left|k-k^{\prime}\right|}\left(1+\frac{d\left(x, \omega ; x^{\prime}, \omega^{\prime}\right)}{2^{-k}+2^{-k^{\prime}}}\right)^{-n-\delta}
$$

The weight function now allows us to give sufficient conditions on a matrix in $\cup_{\omega, k} \Xi_{k}^{\omega} \times \cup_{\omega, k} \Xi_{k}^{\omega}$ so that its associated operator is bounded on $L^{2}\left(\mathbb{R}^{n}\right)$ or is continuous between $L^{2}$ Sobolev spaces. Given a mapping $\chi$ on $S^{*}\left(\mathbb{R}^{n}\right)$, we say that the matrix $b\left(\gamma, \gamma^{\prime}\right)$ belongs to the class $\mathcal{M}_{\delta}^{r}(\chi)$ if

$$
\left|b\left(\gamma, \gamma^{\prime}\right)\right| \leq C_{b} 2^{r k^{\prime}} \mu_{\delta}\left(\gamma, \chi\left(\gamma^{\prime}\right)\right)
$$

where $\chi\left(\gamma^{\prime}\right)=\left(\chi\left(x^{\prime}, \omega^{\prime}\right), k^{\prime}\right)$. We also define $\mathcal{M}^{r}(\chi)=\bigcap_{\delta>0} \mathcal{M}_{\delta}^{r}(\chi)$. An operator $T$ defined as a map from Schwartz class functions on $\mathbb{R}^{n}$ to tempered distributions, is of class $\mathcal{I}^{r}(\chi)$ if its associated matrix in the wave packet frame belongs to $\mathcal{M}^{r}(\chi)$. As a result of Theorem 2.7 in [6] we have that whenever $\chi_{1}, \chi_{2}$ are invertible mappings on $S^{*}\left(\mathbb{R}^{n}\right)$ that satisfy

$$
C^{-1} d\left(x, \omega ; x^{\prime}, \omega^{\prime}\right) \leq d\left(\chi_{l}(x, \omega) ; \chi_{l}\left(x^{\prime}, \omega^{\prime}\right)\right) \leq C d\left(x, \omega ; x^{\prime}, \omega^{\prime}\right), \quad l=1,2
$$

then

$$
\mathcal{I}^{r}\left(\chi_{1}\right): H^{z}\left(\mathbb{R}^{n}\right) \rightarrow H^{z-r}\left(\mathbb{R}^{n}\right) \quad \text { and } \quad \mathcal{I}^{r_{1}}\left(\chi_{1}\right) \circ \mathcal{I}^{r_{2}}\left(\chi_{2}\right) \subset \mathcal{I}^{r_{1}+r_{2}}\left(\chi_{1} \circ \chi_{2}\right) .
$$

3.2. The approximate solution operator. Here we construct an approximate solution operator for $\left(i \partial_{t}+H_{k}^{+}(t, x, D)\right)$ in the sense that when it is composed with $\left(i \partial_{t}+H_{k}^{+}(t, x, D)\right)$ the result is an operator that loses no derivatives. It is then shown that this implies that the operator composed with $\widetilde{P}$ yields a mapping that loses only one derivative. The first portion of the discussion in this subsection actually holds for either $\mathrm{H}^{+}$or $\mathrm{H}^{-}$so we will often drop the superscript when there is no need to distinguish between the two.

Since $H(t, x, \xi)=|\xi| H(t, x, \xi /|\xi|)$ for $|\xi| \geq \frac{3}{4}$ we can redefine $H$ (and similarly $H_{k}$ ) whenever $|\xi|<\frac{3}{4}$ so that $H$ is homogeneous of degree 1 in $\xi$. This redefinition will not effect the results we prove concerning the operators $H(t, x, D), H_{k}(t, x, D)$ as they will almost always be applied to data at frequencies supported away from $B_{\frac{3}{4}}(0)$. Allowing $H$ and $H_{k}$ to be homogeneous of degree one now allows us to take the flow on $T^{*}\left(\mathbb{R}^{n}\right)$, the cotangent bundle on $\mathbb{R}^{n}$, determined by the vector field $\left(-H_{\xi}(t, x, \xi), H_{x}(t, x, \xi)\right)$ and project it down to a flow on the cosphere bundle $S^{*}\left(\mathbb{R}^{n}\right)=\mathbb{R}^{n} \times \mathbb{S}^{n-1}$. 
Given any $\gamma=(x, \omega, k)$, let $\left(x_{\gamma}(s), \omega_{\gamma}(s), \Theta_{\gamma}(s)\right)$ be the solution to

$$
\begin{aligned}
& \frac{d x}{d s}=-\left(H_{k}\right)_{\eta}(s, x, \omega) \\
& \frac{d \omega}{d s}=\left(H_{k}\right)_{x}(s, x, \omega)-\left\langle\omega,\left(H_{k}\right)_{x}(s, x, \omega)\right\rangle \omega \\
& \frac{d \Theta}{d s}=\Theta\left[\omega \cdot\left(H_{k}\right)_{x}(s, x, \omega)^{T}-\left(H_{k}\right)_{x}(s, x, \omega) \cdot \omega^{T}\right]
\end{aligned}
$$

with initial conditions $\left(x_{\gamma}(0), \omega_{\gamma}(0), \Theta_{\gamma}(0)\right)=\left(x_{\gamma}, \omega_{\gamma}, I\right)$. This flow preserves $S^{*}\left(\mathbb{R}^{n}\right) \times O(n)$ and has the property that $\Theta_{\gamma}(t) \omega_{\gamma}(t)=\omega_{\gamma}$ for all time $t$ as $\frac{d}{d t}\left(\Theta_{\gamma}(t) \omega_{\gamma}(t)\right)=0$. Set $\varphi_{\gamma}(s, y)=\varphi_{\gamma}\left(\Theta_{\gamma}(s)\left(y-x_{\gamma}(s)\right)+x_{\gamma}\right)$ and define the operator

$$
\mathbf{e}_{k}(t)\left(\sum_{\gamma} c_{\gamma} \varphi_{\gamma}\right)(y)=\sum_{\gamma: k_{\gamma}=k} c_{\gamma} \varphi_{\gamma}(t, y)
$$

The key result of this section will be to prove that $\sum_{k} \mathbf{e}_{k}(t)$ is indeed an approximate solution operator for $\widetilde{P}$.

Before proceeding, we need to collect a few facts concerning the flows induced by $H$ and $H_{k}$. For each $k \geq 1$, let $\chi_{t}^{k}: S^{*}\left(\mathbb{R}^{n}\right) \rightarrow S^{*}\left(\mathbb{R}^{n}\right)$ denote the mapping $\chi_{t}^{k}(x, \omega)=(x(t), \omega(t))$, where the curves $(x(s), \omega(s))$ are solutions to the first 2 equations in $(3.10)$ with initial values $(x, \omega)$. Also let $\chi_{t}$ be the analogous mapping induced by the nonsmooth Hamiltonian $H$ (that is, the mapping determined by replacing $H_{k}$ by $H$ in $\left.(3.10)\right)$.

First observe that Gronwall's inequality allows us to easily adapt Lemma 2.2 in [6] to our slightly weaker conditions on $H$ and conclude that

$$
C^{-1} d(x, \omega ; \tilde{x}, \tilde{\omega}) \leq d\left(\chi_{t}(x, \omega) ; \chi_{t}(\tilde{x}, \tilde{\omega})\right) \leq C d(x, \omega ; \tilde{x}, \tilde{\omega})
$$

where $C$ is independent of $t \in[-2,2]$. Hence the results of the previously mentioned Theorem 2.7 in [6] apply, and will be used implicitly, in what follows below to show that certain linear transformations are continuous as a maps between Sobolev spaces. that

In the same vein, Lemma 3.6 of [6] is also easily adapted to allow us to conclude

$$
d\left(\chi_{t}^{k}(x, \omega) ; \chi_{t}(x, \omega)\right) \leq C 2^{-k} .
$$

where $C$ is again independent of $t \in[-2,2]$.

Theorem 3.1. Let $\left\{H_{k}(t, x, D)\right\}_{k=1}^{\infty}$ be the family of pseudodifferential operators with symbols $H_{k}(t, \cdot, \cdot) \in S_{1,0}^{1}\left(\mathbb{R}_{x}^{n} \times \mathbb{R}_{\xi}^{n}\right)$ defined above. Then for each fixed $t$ we have

$$
\begin{gathered}
\sum_{k} \mathbf{e}_{k}(t), \sum_{k}\left(i \partial_{t}+H_{k}(t, x, D)\right) \mathbf{e}_{k}(t) \in \mathcal{I}^{0}\left(\chi_{t}\right), \\
\sum_{k} \partial_{t} \circ\left(i \partial_{t}+H_{k}(t, x, D)\right) \mathbf{e}_{k}(t) \in \mathcal{I}^{1}\left(\chi_{t}\right) .
\end{gathered}
$$

Furthermore, if $\tilde{b}\left(t, \gamma^{\prime}, \gamma\right)$ denotes the matrix of $\sum_{k}\left(i \partial_{t}+H_{k}(t, x, D)\right) \mathbf{e}_{k}(t)$, at a fixed time $t$, we then have the following estimates on the matrices for any $t \in[-1,1]$ and 
$N, \delta>0$

$$
\tilde{b}\left(t, \gamma^{\prime}, \gamma\right) \leq \begin{cases}C_{\delta}(1+\varrho(t)) \mu_{\delta}\left(\gamma^{\prime}, \chi_{t}(\gamma)\right) & k_{\gamma^{\prime}} \geq k_{\gamma}-2 \\ C_{\delta, N}(1+\varrho(t)) 2^{-k_{\gamma} N} \mu_{\delta}\left(\gamma^{\prime}, \chi_{t}(\gamma)\right) & k_{\gamma^{\prime}} \leq k_{\gamma}-3\end{cases}
$$

The constants $C_{\delta}, C_{\delta, N}$ are independent of $t \in[-2,2]$.

Proof. Since the claim $\sum_{k} \mathbf{e}_{k}(t) \in \mathcal{I}^{0}\left(\chi_{t}\right)$ follows as in Theorem 3.2 of [6], we focus on proving the other results. Consider the operator $i \partial_{s}+H_{k}(s, x, D)$ applied to $\varphi_{\gamma}(s, y)$ and abbreviate the integral curve $\left(x_{\gamma}(s), \omega_{\gamma}(s), \Theta_{\gamma}(s)\right)$ defining the function by $\left(x_{s}, \omega_{s}, \Theta_{s}\right)$. A straightforward computation shows that

$$
\partial_{s} \varphi_{\gamma}(s, y)=L\left(s, x_{s}, \omega_{s}, y, \partial_{y}\right) \varphi_{\gamma}(s, y)
$$

where

$$
\begin{array}{r}
L\left(s, x, \omega, y, \partial_{y}\right)=\left\langle\left(H_{k}\right)_{\eta}(s, x, \omega), \partial_{y}\right\rangle+\left\langle\left(H_{k}\right)_{x}(s, x, \omega), y-x\right\rangle\left\langle\omega, \partial_{y}\right\rangle \\
-\langle\omega, y-x\rangle\left\langle\left(H_{k}\right)_{x}(s, x, \omega), \partial_{y}\right\rangle
\end{array}
$$

By employing a Taylor expansion of $H_{k}(s, y, \eta)$ about the point $\left(x_{s},|\eta| \omega_{s}\right)$ with second order error term $R(\gamma, s, y, \eta)$ it is not hard to see that the operator

$$
i L\left(s, x_{s}, \omega_{s}, y, \partial_{y}\right)+\left(H_{k}\right)(s, y, D)
$$

has the symbol

$$
\begin{aligned}
& \left\langle\left(H_{k}\right)_{x}\left(s, x_{s}, \omega_{s}\right), y-x_{s}\right\rangle|\eta|\left(\left\langle\omega_{s}, \omega_{s}-\eta /|\eta|\right\rangle\right) \\
& \quad+\left\langle\omega_{s}, y-x_{s}\right\rangle\left\langle\left(H_{k}\right)_{x}\left(s, x_{s}, \omega_{s}\right), \eta\right\rangle+R(\gamma, s, y, \eta)
\end{aligned}
$$

Call the operator determined by the first 2 terms in the sum $T_{\gamma, s}$, and let $\partial_{s} T_{\gamma, s}$ be the operator obtained by differentiating its symbol with respect to $s$. Also, abbreviate the operator determined by $R(\gamma, s, \cdot)$ by $R_{\gamma, s}$ and take a similar convention for $\partial_{s} R_{\gamma, s}$. By using the Fourier transform we see that for $l=0,1$, $\int\left(\partial_{s}^{l} T_{\gamma, s} \varphi_{\gamma}\right)(s, y) \overline{\varphi_{\tilde{\gamma}}(y)} d y$ vanishes unless both $\left|\omega_{\tilde{\gamma}}-\omega_{s}\right| \leq 2^{-k+4}$ and $\left|k_{\gamma}-k_{\tilde{\gamma}}\right| \leq 1$. Using standard estimates on the spatial localization of the wave packets we have that for any $\delta>0$ there exists $C_{\delta}$ such that

$$
\left|\int\left(\partial_{s}^{l} T_{\gamma, s}\right) \varphi_{\gamma} \overline{\varphi_{\tilde{\gamma}}} d y\right| \leq C_{\delta} 2^{k l} \mu_{\delta}\left(\chi_{s}^{k}(\gamma), \tilde{\gamma}\right) \approx C_{\delta} 2^{k l} \mu_{\delta}\left(\chi_{s}(\gamma), \tilde{\gamma}\right)
$$

with the latter approximation following from (3.12).

Now let $F_{11}^{\alpha}, F_{22}^{\alpha}, F_{12}^{i j}$ be the unique homogeneous symbols of degree 0 such that

$$
\begin{aligned}
R(\gamma, s, y, \eta)=\sum_{|\alpha|=2}\left(y-x_{s}\right)^{\alpha}|\eta| F_{11}^{\alpha}(\gamma, s, y, \eta) & +\sum_{|\alpha|=2}\left(\eta-|\eta| \omega_{s}\right)^{\alpha}|\eta|^{-1} F_{22}^{\alpha}(\gamma, s, y, \eta) \\
& +\sum_{1 \leq i, j \leq n}\left(y-x_{s}\right)_{i}\left(\eta-|\eta| \omega_{s}\right)_{j} F_{12}^{i j}(\gamma, s, y, \eta) .
\end{aligned}
$$

Also, observe that (3.5) implies the following estimates on $F=F_{11}^{\alpha}, F_{22}^{\alpha}$, or $F_{12}^{i j}$ for $\eta \in \operatorname{supp}\left(\varphi_{\gamma}\right)$

$$
\left|\partial_{s}^{j} \partial_{y}^{\beta_{1}} \partial_{\eta}^{\beta_{2}} F(\gamma, s, y, \eta)\right| \leq C_{\beta_{1}, \beta_{2}}(\varrho(s)+1) 2^{\frac{k}{2}\left(\left|\beta_{1}\right|+j\right)}|\eta|^{-\left|\beta_{2}\right|} .
$$

Writing

$$
\partial_{s}\left(\left(T_{\gamma, s}+R_{\gamma, s}\right) \varphi_{\gamma}(s, \cdot)\right)=\left(\partial_{s} T_{\gamma, s}+\partial_{s} R_{\gamma, s}\right) \varphi_{\gamma}(s, \cdot)+\left(T_{\gamma, s}+R_{\gamma, s}\right) \partial_{s} \varphi_{\gamma}(s, \cdot)
$$


we see that the theorem is now a consequence of $(3.15),(3.13)$, and the following Lemma.

Lemma 3.2. Let $B(y, \eta) \in S_{1, \frac{1}{2}}^{l}$ with symbol estimates of the form

$$
\left|\partial_{y}^{\beta_{1}} \partial_{\eta}^{\beta_{2}} B(y, \eta)\right| \leq M \cdot C_{\beta_{1}, \beta_{2}}\langle\eta\rangle^{l+\frac{\left|\beta_{1}\right|}{2}-\left|\beta_{2}\right|}
$$

where $M>0$ is some constant independent of $\beta_{1}, \beta_{2}$. Suppose also that $\psi_{\gamma}$ is a function of the form

$$
\begin{aligned}
& \widehat{\psi_{\gamma}}(\xi)=e^{-i\left\langle x_{\gamma}, \xi\right\rangle} 2^{k\left(i-j+\frac{\left|\beta_{1}\right|}{2}-\frac{\left|\beta_{2}\right|}{2}-l+\frac{\left|\beta_{3}\right|}{2}-\frac{(n+1)}{4}\right)} . \\
& \left\langle\omega_{\gamma}, \partial_{\xi}\right\rangle^{i}\left\langle\omega_{\gamma}^{\perp}, \partial_{\xi}\right\rangle^{\beta_{1}}\left\langle\omega_{\gamma}, \xi\right\rangle^{j}\left\langle\omega_{\gamma}^{\perp}, \xi\right\rangle^{\beta_{2}}|\xi|^{l}\left(\xi-|\xi| \omega_{\gamma}\right)^{\beta_{3}} h_{k}^{\omega_{\gamma}}(\xi) .
\end{aligned}
$$

Then for $\gamma=\left(x_{\gamma}, \omega_{\gamma}, k_{\gamma}\right), \tilde{\gamma}=\left(x_{\tilde{\gamma}}, \omega_{\tilde{\gamma}}, k_{\tilde{\gamma}}\right)$ we have the following inequality:

$$
\left|\int \overline{\varphi_{\tilde{\gamma}}(y)} B(y, D) \psi_{\gamma}(y) d y\right| \leq \begin{cases}M \cdot C_{\delta} 2^{k_{\gamma} l} \mu_{\delta}(\gamma, \tilde{\gamma}) & \left|k_{\gamma}-k_{\tilde{\gamma}}\right| \leq 2 \\ M \cdot C_{\delta, N} 2^{-\min \left(k_{\gamma}, k_{\tilde{\gamma}}\right) N} \mu_{\delta}(\gamma, \tilde{\gamma}) & \left|k_{\gamma}-k_{\tilde{\gamma}}\right| \geq 3\end{cases}
$$

where $C_{\delta}, C_{\delta, N}$ depend on $\delta, N$, the constants $C_{\beta_{1}, \beta_{2}}$, and on the sequence of indices taken in (3.16), but not on $M$ or the choice of $\gamma, \tilde{\gamma}$.

Proof. For the purposes of this proof, given a sequence $K$ of indices and/or quantities, $C_{K}$ will denote some constant depending only on $K$ and the constants $C_{\beta_{1}, \beta_{2}}$. We will also often abbreviate $k_{\gamma}$ by $k$.

Integrating by parts in the integral defining $B(y, D) \psi_{\gamma}(y)$ yields estimates of the form

$$
\begin{aligned}
\left|\left\langle\omega_{\gamma}, y-x_{\gamma}\right\rangle^{j}\left(y-x_{\gamma}\right)^{\alpha_{1}}\left\langle\omega_{\gamma}^{\perp}, \partial_{y}\right\rangle^{\alpha_{2}} \partial_{y}^{\alpha_{3}} B(y, D) \psi_{\gamma}(y)\right| \\
\quad \leq M C_{j, \alpha_{1}, \alpha_{2}, \alpha_{3}} 2^{\frac{k(n+1)}{4}+k l+\frac{k}{2}\left(\left|\alpha_{2}\right|+2\left|\alpha_{3}\right|-\left|\alpha_{1}\right|-2 j\right)}
\end{aligned}
$$

where $C_{j, \alpha_{1}, \alpha_{2}, \alpha_{3}}$ is independent of $M$. This shows that such the function is concentrated in space to a rectangle of width $2^{k}$ in the $\omega_{\gamma}$ direction and width $2^{\frac{k}{2}}$ orthogonally. Let $B \psi_{\gamma}$ abbreviate $B(\cdot, D) \psi_{\gamma}$. As a result of the estimates above, integration by parts in the integral defining $\widehat{B \psi_{\gamma}}(\xi)$ gives us

$$
\left|\widehat{B \psi_{\gamma}}(\xi)\right| \leq M C_{N} 2^{k l-\frac{k(n+1)}{4}}\left(1+2^{-k}\left|\pi_{\gamma}^{\perp}(\xi)\right|^{2}+2^{-2 k}|\xi|^{2}\right)^{-N}
$$

where $\pi_{\gamma}^{\perp}$ denotes projection on to the subspace orthogonal to $\omega_{\gamma}$. Hence $\widehat{B \psi_{\gamma}}(\xi)$ is concentrated in a rectangle of width $2^{\frac{k}{2}}$ in directions orthogonal to $\omega_{\gamma}$ and length $2^{k}$ in directions parallel to $\omega_{\gamma}$.

Another important estimate on $\widehat{B \psi_{\gamma}}(\xi)$ is obtained through integration by parts with respect to $\eta$ and $y$ in the integral

$$
\widehat{B \psi_{\gamma}}(\xi)=\iint e^{i\langle y, \eta-\xi\rangle} B(y, \eta) \widehat{\psi_{\gamma}}(\eta) d \eta d y
$$

This allows us to obtain the following estimates for any $N$

$$
\left|\widehat{B \psi}_{\gamma}(\xi)\right| \leq M C_{N} 2^{k l-\frac{3 k(n+1)}{4}} \int_{\operatorname{supp}\left(\widehat{\varphi}_{\gamma}\right)}\left(1+2^{-k}|\xi-\eta|^{2}\right)^{-N} d \eta
$$


When $\left|k_{\gamma}-k_{\tilde{\gamma}}\right| \leq 2$ and $\left\langle\omega_{\gamma}, \omega_{\tilde{\gamma}}\right\rangle \geq-\frac{1}{2}$ we use the inequalities (3.19) after observing that for $\xi \in \operatorname{supp}\left(\widehat{\varphi}_{\tilde{\gamma}}\right)$ we have

$$
\left|\pi_{\gamma}^{\perp}(\xi)\right| \geq c\left(2^{k}\left|\omega_{\gamma}-\omega_{\tilde{\gamma}}\right|-2^{\frac{k}{2}}\right)
$$

with $c$ uniform over all such $\gamma, \tilde{\gamma}$. This yields

$$
\begin{array}{r}
\left|\int \widehat{B \psi_{\gamma}} \widehat{\widehat{\varphi} \tilde{\gamma}}\right| \leq M C_{N} 2^{-\frac{k(n+1)}{2}+k l} \int_{\operatorname{supp}\left(\hat{\varphi}_{\tilde{\gamma}}\right)}\left(1+2^{-k}\left|\left\langle\omega_{\gamma}, \xi\right\rangle\right|+2^{-\frac{k}{2}}\left|\pi_{\gamma}^{\perp}(\xi)\right|\right)^{-2 N} d \xi \\
\leq M C_{N}\left(1+2^{k}\left|\omega_{\gamma}-\omega_{\tilde{\gamma}}\right|^{2}\right)^{-N} .
\end{array}
$$

Now consider the case where either (a) $\left|k_{\gamma}-k_{\tilde{\gamma}}\right| \leq 2$ with $\left\langle\omega_{\tilde{\gamma}}, \omega_{\gamma}\right\rangle \leq-\frac{1}{2}$ or (b) $\left|k_{\gamma}-k_{\tilde{\gamma}}\right| \geq 3$ with no restriction on $\omega_{\gamma}, \omega_{\tilde{\gamma}}$. In this case, there exists $c$ independent of choice of such $\gamma, \tilde{\gamma}$ such that $|\xi-\eta| \geq c 2^{\max \left(k_{\gamma}, k_{\tilde{\gamma}}\right)}$ for $\xi \in \operatorname{supp}\left(\varphi_{\tilde{\gamma}}\right), \eta \in \operatorname{supp}\left(\varphi_{\tilde{\gamma}}\right)$. We can thus apply (3.21) to get

$$
\left|\int \widehat{B \psi_{\gamma}} \overline{\widehat{\varphi}_{\tilde{\gamma}}} d \xi\right| \leq M \tilde{C}_{N} 2^{\max \left(k_{\gamma}, k_{\tilde{\gamma}}\right)(n+1)+k_{\gamma} l}\left(1+2^{\max \left(k_{\gamma}, k_{\tilde{\gamma}}\right.}\right)^{-N}
$$

These inequalities, along with the results established in the previous case, yield the following estimates without restriction on $\gamma, \tilde{\gamma}$

$$
\left|\int \overline{\varphi_{\tilde{\gamma}}} B \psi_{\gamma}\right| \leq M C_{N} \zeta\left(N, k_{\tilde{\gamma}}, k_{\gamma}\right) 2^{k_{\gamma} l-\left|k_{\gamma}-k_{\tilde{\gamma}}\right| N}\left(1+2^{k}\left|\omega_{\gamma}-\omega_{\tilde{\gamma}}\right|^{2}\right)^{-N}
$$

where

$$
\zeta\left(N, k_{\tilde{\gamma}}, k_{\gamma}\right) \leq \begin{cases}2^{-k_{\tilde{\gamma}} N} & k_{\tilde{\gamma}} \geq k_{\gamma}+3 \\ 1 & \left|k_{\tilde{\gamma}}-k_{\gamma}\right| \leq 2 \\ 2^{-k_{\gamma} N} & k_{\tilde{\gamma}} \leq k_{\gamma}-3\end{cases}
$$

To obtain the conclusion of the lemma when $\left|k_{\tilde{\gamma}}-k_{\gamma}\right| \leq 2$, we incorporate the estimates (3.18) on $B \psi_{\gamma}$ as well as the standard inequalities (3.7) on $\varphi_{\tilde{\gamma}}$ to get that

$$
\left(1+2^{k}\left|\omega_{\gamma}-\omega_{\tilde{\gamma}}\right|^{2}\right)^{-2 N}\left|\int \overline{\varphi_{\tilde{\gamma}}} B \psi_{\gamma}\right| \leq M C_{N} 2^{k l}\left(1+\frac{d\left(x_{\gamma}, \omega_{\gamma}, x_{\tilde{\gamma}}, \omega_{\tilde{\gamma}}\right)}{2^{-k}+2^{-k_{\tilde{\gamma}}}}\right)^{-N} .
$$

The result follows for $\left|k_{\tilde{\gamma}}-k_{\gamma}\right| \leq 2$ by observing that this implies

$$
\begin{aligned}
\left|\int \frac{\bar{\varphi} \tilde{\gamma}}{B} \psi_{\gamma}\right|^{2} & \leq C_{N} 2^{2 k l} M^{2} 2^{-2\left|k_{\gamma}-k_{\tilde{\gamma}}\right| N}\left(1+2^{k+1}\left|\omega_{\gamma}-\omega_{\tilde{\gamma}}\right|^{2}\right)^{-2 N}\left|\int \overline{\varphi_{\tilde{\gamma}}} B \psi_{\gamma}\right| \\
& \leq C_{N} 2^{2 k l} M^{2} \mu_{N-n}(\gamma, \tilde{\gamma})^{2}
\end{aligned}
$$

A similar calculation establishes the desired estimate when $\left|k_{\tilde{\gamma}}-k_{\gamma}\right| \geq 3$.

We are now able to show the following:

Theorem 3.3. Suppose $H_{k}=H_{k}^{+}$so that $\mathbf{e}_{k}(t)$ is defined by the integral curves of $H_{k}^{+}$. Then

$$
\sum_{k}\left(i \partial_{t}+H_{k}^{-}(t, x, D)\right) \circ\left(i \partial_{t}+H_{k}^{+}(t, x, D)\right) \mathbf{e}_{k}(t) \in \mathcal{I}^{1}\left(\chi_{t}\right) .
$$

Proof. As a consequence of Theorem 3.1 we have that

$$
\sum_{k} i \partial_{t} \circ\left(i \partial_{t}+H_{k}^{+}(t, x, D)\right) \mathbf{e}_{k}(t) \in \mathcal{I}^{1}\left(\chi_{t}\right)
$$


So it remains to show that

$$
\sum_{k} H_{k}^{-}(t, x, D) \circ\left(i \partial_{t}+H_{k}^{+}(t, x, D)\right) \mathbf{e}_{k}(t) \in \mathcal{I}^{1}\left(\chi_{t}\right) .
$$

Let $\tilde{w}_{k}(\tilde{\gamma}, \gamma), w_{k}(\tilde{\gamma}, \gamma)$ denote the matrices associated to $H_{k}^{-}(t, x, D)$ and $\left(i \partial_{t}+\right.$ $H_{k}^{+}(t, x, D) \mathbf{e}_{k}(t)$ respectively. Our strategy will be to show that for any 3 triples $\tilde{\gamma}, \gamma, \gamma^{\prime}$ with $\left|k_{\gamma^{\prime}}-k\right| \leq 1$ and $\delta>0$ that there exists $C_{\delta}$ independent of choice of triples such that

$$
\left|\tilde{w}_{k}(\tilde{\gamma}, \gamma) w_{k}\left(\gamma, \gamma^{\prime}\right)\right| \leq C_{\delta} 2^{k_{\gamma}} \mu_{\delta}(\tilde{\gamma}, \gamma) \mu_{\delta}\left(\gamma, \chi_{t}\left(\gamma^{\prime}\right)\right)
$$

The theorem then follows by Lemma 2.5 and Theorem 2.7 in [6].

When $k_{\gamma} \geq k-2$,

$$
H_{k}^{-}(s, y, \eta)\left(\beta_{k_{\gamma}-1}(\eta)+\beta_{k_{\gamma}}(\eta)+\beta_{k_{\gamma}+1}(\eta)\right)
$$

is a symbol of order $S_{1, \frac{1}{2}}^{1}$, with symbol estimates that can be taken to be uniform in $k$. The desired inequalities are then a result of Lemma 3.2 and Theorem 3.1.

The case $k_{\gamma}<k-2$ is more involved. Integration by parts in the integral

$$
\left(T_{k} \varphi_{\gamma}\right)(y):=2^{-\frac{k(n+1)}{4}} \int e^{i\langle y-x, \eta\rangle} H_{k}^{-}(s, y, \eta) h_{k_{\gamma}}^{\omega}(\eta) d \eta
$$

yields the estimates

$$
\left|\partial_{y}^{\alpha} T_{k} \varphi_{\gamma}(y)\right| \leq C_{\alpha, N} 2^{k_{\gamma}+\frac{k_{\gamma}(n+1)}{4}+\max \left(k_{\gamma}, \frac{k}{2}\right)|\alpha|}\left(1+2^{k_{\gamma}}|\langle\omega, y-x\rangle|+2^{k_{\gamma}}|y-x|^{2}\right)^{-N}
$$

This result, along with the estimates on the $\widehat{T_{k} \varphi_{\gamma}}$ it induces, gives us the following matrix estimates which are much weaker than that of Lemma 3.2

$$
\left|\int\left(T_{k} \varphi_{\gamma}\right) \overline{\varphi_{\tilde{\gamma}}}\right| \leq C_{\delta} 2^{k(2 \delta+2 n+3)} \mu_{\delta}(\tilde{\gamma}, \gamma)
$$

However, due to the estimates of Theorem 3.1 when $k_{\gamma}<k-2$ we have

$$
\left|\tilde{w}_{k}(\tilde{\gamma}, \gamma) w_{k}\left(\gamma, \gamma^{\prime}\right)\right| \leq C_{\delta} 2^{k_{\gamma}}\left(2^{k(2 \delta+2 n+3)} \mu_{\delta}(\tilde{\gamma}, \gamma)\right)\left(2^{-k(2 \delta-2 n-3)} \mu_{\delta}\left(\gamma, \chi_{t}(\gamma)^{\prime}\right)\right)
$$

As noted above, this completes the proof.

We are now able to show that $\sum_{k} \mathbf{e}_{k}(t)$ is indeed an approximate solution operator for $\widetilde{P}$.

Theorem 3.4. Let $\mathbf{e}_{k}(t)$ be defined by the integral curves of $H_{k}^{+}$as in Theorem 3.3. Then for $-1 \leq z \leq 1$,

$$
\sum_{k} \widetilde{P}(t, x, D) \mathbf{e}_{k}(t): H^{z+1}\left(\mathbb{R}^{n}\right) \rightarrow H^{z}\left(\mathbb{R}^{n}\right),
$$

with operator norm bounded by $C(\varrho(t)+1)$ for $t \in[-2,2]$.

Proof. Begin by writing $\sum_{k} \widetilde{P}(t, x, D) \mathbf{e}_{k}(t)$ as

$$
\begin{aligned}
\sum_{k} \widetilde{P}(t, x, D) \mathbf{e}_{k}(t)=\sum_{k} Q^{k}(t, x, D) \mathbf{e}_{k}(t) & +\left(\sum_{2}^{n} \tilde{b}_{i}\left(t, x^{\prime}\right) \partial_{i}+\tilde{b}_{1}\left(t, x^{\prime}\right) \partial_{t}\right) \circ \sum_{k} \mathbf{e}_{k}(t) \\
& \left.+\sum_{k}(Q .23) \quad(t, x, D)-Q^{k}(t, x, D)\right) \mathbf{e}_{k}(t)
\end{aligned}
$$


We begin by considering the first term here. By equation (3.3) and Theorem 3.3 it suffices to control the sum $\sum_{k} E_{k}(t, x, D) \mathbf{e}_{k}(t)$ where

$$
\begin{aligned}
E_{k}^{+}(t, x, D)=-i\left(\partial_{t} H_{k}^{+}\right)(t, x, D) & -i\left(\partial_{t} H_{k}^{-}\right)(t, x, D) \\
& +\left(H_{k}^{+} \circ H_{k}^{-}\right)(t, x, D)-\left(H_{k}^{+} H_{k}^{-}\right)(t, x, D) .
\end{aligned}
$$

Here the symbol of $\left(\partial_{t} H_{k}^{ \pm}\right)(t, x, D)$ is $\left(\partial_{t} H_{k}^{ \pm}\right)(t, x, \xi)$ and the symbol of the operator $\left(H_{k}^{+} H_{k}^{-}\right)(t, x, D)$ is $H_{k}^{+}(t, x, \xi) H_{k}^{-}(t, x, \xi)$. By splitting the sum up into groups of integers that are equal modulo 4 , it suffices to show that the following sum is a map that loses one derivative:

$$
\sum_{k} E_{4 k}(t, x, D) \mathbf{e}_{4 k}(t)=\left(\sum_{k} E_{4 k}(t, x, D)\left(\beta_{4 k-1}+\beta_{4 k}+\beta_{4 k+1}\right)\right) \circ\left(\sum_{k} \mathbf{e}_{4 k}(t)\right) .
$$

Employing the first claim in Theorem 3.1, the sum is now handled by observing that by examining the symbol of (3.24)

$$
\sum_{k} E_{4 k}(t, x, D)\left(\beta_{4 k-1}+\beta_{4 k}+\beta_{4 k+1}\right) \in S_{1, \frac{1}{2}}^{1}
$$

with symbol estimates bounded by $C(\varrho(t)+1)$.

Next observe that since $b_{i}(t, \cdot) \in \operatorname{Lip}\left(\mathbb{R}^{n}\right)$ with Lipschitz norm bounded by $C(\varrho(t)+1)$ the second term in (3.23) also has the desired mapping properties, as (3.13) permits a straightforward proof that $\sum_{k} \partial_{t} \circ \mathbf{e}_{k}(t) \in \mathcal{I}^{1}\left(\chi_{t}\right)$.

Controlling the last term in (3.23) reduces to showing that if $a=\tilde{a}_{i j}$ for some $i, j$ or $a=\tilde{\rho}$, then the sum

$$
\sum_{k}\left(a\left(t, x^{\prime}\right)-a^{k}\left(t, x^{\prime}\right)\right) \beta_{k}(D): H^{z-1}\left(\mathbb{R}^{n}\right) \rightarrow H^{z}\left(\mathbb{R}^{n}\right)
$$

for $-1 \leq z \leq 1$. However, this result follows by the same methods used in Theorem 4.5 of [6] except that in our case the norm of this operator is now bounded by $C(\varrho(t)+1)$ for some uniform constant $C$.

We pause to remark that if $\mathbf{e}_{k}(t)$ is instead defined by the integral curves of $H_{k}^{-}$, then a symmetric line of reasoning shows that it is also an approximate solution operator for $\widetilde{P}$. This fact will be used in the next section.

3.3. Representing the solution. Here we define operators $\mathbf{c}_{k}(t, s), \mathbf{s}_{k}(t, s)$ as in section 4 of [6]. For a given $\gamma=\left(x_{\gamma}, \omega_{\gamma}, k\right)$, let $\left(x_{\gamma}^{ \pm}(t, s), \omega_{\gamma}^{ \pm}(t, s), \Theta_{\gamma}^{ \pm}(t, s)\right)$ be the solution to

$$
\begin{aligned}
\frac{d x^{ \pm}}{d t} & =-\left(H_{k}^{ \pm}\right)_{\eta}(t, x, \omega) \\
\frac{d \omega^{ \pm}}{d t} & =\left(H_{k}^{ \pm}\right)_{x}(t, x, \omega)-\left\langle\omega,\left(H_{k}^{ \pm}\right)_{x}(t, x, \omega)\right\rangle \omega \\
\frac{d \Theta^{ \pm}}{d t} & =\Theta\left[\omega \cdot\left(H_{k}^{ \pm}\right)_{x}(t, x, \omega)^{T}-\left(H_{k}^{ \pm}\right)_{x}(t, x, \omega) \cdot \omega^{T}\right]
\end{aligned}
$$


with the initial conditions $\left.\left(x_{\gamma}^{ \pm}(t, s), \omega_{\gamma}^{ \pm}(t, s), \Theta_{\gamma}^{ \pm}(t, s)\right)\right|_{t=s}=\left(x_{\gamma}, \omega_{\gamma}, I\right)$. For each $\gamma$ with $k_{\gamma}>0$, define the function $\vartheta_{\gamma}$ by $\widehat{\vartheta}_{\gamma}(\xi)=-i 2^{k}\left\langle\omega_{\gamma}, \xi\right\rangle^{-1} \widehat{\varphi}_{\gamma}(\xi)$. We now set

$$
\begin{aligned}
& \varphi_{\gamma}^{ \pm}(t, s, y)=\varphi_{\gamma}\left(\Theta_{\gamma}^{ \pm}(t, s)\left(y-x_{\gamma}^{ \pm}(t, s)\right)+x_{\gamma}\right) \\
& \vartheta_{\gamma}^{ \pm}(t, s, y)=\frac{2}{H_{k}^{+}\left(s, x_{\gamma}, \omega_{\gamma}\right)-H_{k}^{-}\left(s, x_{\gamma}, \omega_{\gamma}\right)} \vartheta_{\gamma}\left(\Theta_{\gamma}^{ \pm}(t, s)\left(y-x_{\gamma}^{ \pm}(t, s)\right)+x_{\gamma}\right) .
\end{aligned}
$$

This leads us to define the operators $\mathbf{c}_{k}(t, s), \mathbf{s}_{k}(t, s)$ on a function $f=\sum_{\gamma} c_{\gamma} \varphi_{\gamma}$ by

$$
\begin{aligned}
& \left(\mathbf{c}_{k}(t, s) f\right)(y)=\frac{1}{2} \sum_{k_{\gamma}=k} c_{\gamma}\left(\varphi_{\gamma}^{+}(t, s, y)+\varphi_{\gamma}^{-}(t, s, y)\right) \\
& \left(\mathbf{s}_{k}(t, s) f\right)(y)=\frac{1}{2} \sum_{k_{\gamma}=k} 2^{-k} c_{\gamma}\left(\vartheta_{\gamma}^{+}(t, s, y)-\vartheta_{\gamma}^{-}(t, s, y)\right)
\end{aligned}
$$

Now set $\tilde{\mathbf{c}}(t, s)=\sum_{k=0}^{\infty} \mathbf{c}_{k}(t, s)$ and $\tilde{\mathbf{s}}(t, s)=\sum_{k \geq k_{0}} \mathbf{s}_{k}(t, s)+(t-s) \sum_{k<k_{0}} \Delta_{k}$ where $\Delta_{k}$ is defined by

$$
\Delta_{k}\left(\sum_{\gamma} c_{\gamma} \varphi_{\gamma}\right)=\sum_{k_{\gamma}=k} c_{\gamma} \varphi_{\gamma}
$$

The proof Lemma 4.4 of [6] uses only the fact that $\left\|\left(H_{k}^{ \pm}\right)_{x}(\cdot, \cdot, \xi)\right\|_{L_{t, x}^{\infty}} \leq C$ for some $C$ independent of choice of $\xi \in \mathbb{S}^{n-1}$ and frequency index $k$ and is easily adapted to our circumstances. It states the following:

Lemma 3.5. For $k_{0}$ sufficiently large and depending on the estimates on the coefficients $\left\{a_{i j}(t, x)\right\}_{i j}$, the operator $\left.\partial_{t} \tilde{\mathbf{s}}(t, s)\right|_{t=s}$ admits a bounded inverse on $L^{2}\left(\mathbb{R}^{n}\right)$. This inverse extends to a bounded operator on $H^{z}\left(\mathbb{R}^{n}\right)$ and is continuous in $s$ in the norm topology on $H^{z}\left(\mathbb{R}^{n}\right)$.

We thus choose $k_{0}$ to be as large as required in the lemma and set

$$
\mathbf{s}(t, s)=\tilde{\mathbf{s}}(t, s) \circ\left(\left.\partial_{t} \tilde{\mathbf{s}}(t, s)\right|_{t=s}\right)^{-1} .
$$

Also, define

$$
\mathbf{c}(t, s)=\tilde{\mathbf{c}}(t, s)-\mathbf{s}(t, s) \circ\left(\left.\partial_{t} \tilde{\mathbf{c}}(t, s)\right|_{t=s}\right) .
$$

Therefore, $\mathbf{c}$ is an operator that loses no derivatives, $\mathbf{s}$ is an operator that gains 1 derivative, and the following properties hold

$$
\begin{array}{lll}
\left.\mathbf{c}(t, s)\right|_{t=s}=I, & \left.\partial_{t} \mathbf{c}(t, s)\right|_{t=s}=0, \\
\left.\mathbf{s}(t, s)\right|_{t=s}=0, & \left.\partial_{t} \mathbf{s}(t, s)\right|_{t=s}=I .
\end{array}
$$

We now define operators $T_{0}, T_{1}$ by

$$
T_{0}(t, s)=P(t, x, D) \mathbf{c}(t, s) \quad T_{1}(t, s)=P(t, x, D) \mathbf{s}(t, s) .
$$

A proof similar to that in Theorem 3.4 now shows the following:

Theorem 3.6. $T_{0}(t, s), T_{1}(t, s)$ enjoy the following mapping properties for $-1 \leq$ $z \leq 2$

$$
\begin{aligned}
& T_{0}(t, s): H^{z+1}\left(\mathbb{R}^{n}\right) \rightarrow H^{z}\left(\mathbb{R}^{n}\right) \\
& T_{1}(t, s): H^{z}\left(\mathbb{R}^{n}\right) \rightarrow H^{z}\left(\mathbb{R}^{n}\right)
\end{aligned}
$$

with operator norm bounded by $C(\varrho(t)+1)$ for $t \in[-2,2]$.

We are now able to represent solutions to hyperbolic pseudodifferential equations as desired. 
Theorem 3.7. Suppose $w_{0} \in H^{z+1}\left(\mathbb{R}^{n}\right), w_{1} \in H^{z}\left(\mathbb{R}^{n}\right), F \in L_{t}^{1}\left([-2,2] ; H^{z}\left(\mathbb{R}^{n}\right)\right)$ for some $-1 \leq z \leq 1$. Then there exists $G \in L_{t}^{1}\left([-2,2] ; H^{z}\right)$ with norm bounded by

$$
\|G\|_{L_{t}^{1}\left([-2,2] ; H^{z}\right)} \leq C\left(\left\|w_{0}\right\|_{H^{z+1}}+\left\|w_{1}\right\|_{H^{z}}+\|F\|_{L_{t}^{1}\left([-2,2] ; H^{z}\right)}\right)
$$

such that

$$
w(t, x)=\left(\mathbf{c}(t, 0) w_{0}\right)(x)+\left(\mathbf{s}(t, 0) w_{1}\right)(x)+\int_{0}^{t}(\mathbf{s}(t, s) F(s, \cdot))(x) d s
$$

is a solution to the Cauchy Problem

$$
\begin{aligned}
\left.w(t, x)\right|_{t=0} & =w_{0}(x) \\
\left.\partial_{t} w(t, x)\right|_{t=0} & =w_{1}(x) \\
(\widetilde{P}(t, x, D) w)(t, x) & =F(t, x)
\end{aligned}
$$

Proof. This is essentially the same proof as in Theorem 4.6 of [6]. As in that Theorem, we are lead to solve the integral equation

$$
G(t, x)+\int_{0}^{t} T_{1}(t, s) G(s, x) d s=\tilde{F}(t, x) .
$$

where $\tilde{F}(t, x)=F(t, x)-\left(T_{0}(t, 0) w_{0}\right)(x)+\left(T_{1}(t, 0) w_{1}\right)(x)$.

In order to solve this equation set

$$
G_{n}(t, x)=\int_{0}^{t} \int_{0}^{s_{1}} \cdots \int_{0}^{s_{n-1}} T_{1}\left(t, s_{1}\right) T_{1}\left(s_{1}, s_{2}\right) \cdots T_{1}\left(s_{n-1}, s_{n}\right) \tilde{F}\left(s_{n}, x\right) d s_{n} \cdots d s_{1}
$$

and $G(t, x)=\sum_{n=1}(-1)^{n} G_{n}(t, x)+\tilde{F}(t, x)$. As before, this sum converges absolutely in $L_{t}^{1}\left([-2,2] ; H^{z}\right)$ to a solution to the integral equation as a result of the more complicated estimate

$$
\begin{aligned}
& \left\|G_{n}(t, \cdot)\right\|_{H^{z}} \\
\leq & \int_{0}^{t} \int_{0}^{s_{1}} \cdots \int_{0}^{s_{n-1}} C^{n}(\varrho(t)+1)\left(\varrho\left(s_{1}\right)+1\right) \cdots\left(\varrho\left(s_{n-1}\right)+1\right)\left\|\tilde{F}\left(s_{n}, \cdot\right)\right\|_{H^{z}} d s_{n} \cdots d s_{1} \\
& \leq\|\tilde{F}\|_{L_{t}^{1}\left([-2,2] ; H^{z}\left(\mathbb{R}^{n}\right)\right)}(\varrho(t)+1) C^{n}\left(1+\|\varrho\|_{L^{1}}\right)^{n-1} /(n-1) !
\end{aligned}
$$

\section{Estimates Via the Parametrix}

We now have the tools to represent solutions $w$ to the wave equation over the domain $\mathbb{R} \times(-2,2) \times \mathbb{R}^{n-1}$ whose partial Fourier transform in $t, x^{\prime}$ is supported in the set

$$
\operatorname{supp}\left(\widehat{w}\left(\tau, x_{1}, \xi^{\prime}\right)\right) \subset\left\{\left(\tau, \xi^{\prime}\right): \tau>11\left|\xi^{\prime}\right|\right\} \backslash B_{4}(0)
$$

In this section, given $s, p, q$ as in Theorem 1.1 we will thus discuss how to show the following estimate for $w$ that can be represented by the wave packet construction of Section 3 and enjoys the property (4.1):

$$
\|w\|_{L_{t}^{p} L_{x}^{q}\left([-1,1] \times(-2,2) \times \mathbb{R}_{x^{\prime}}^{n-1}\right)} \lesssim\left\|w_{0}\right\|_{H_{t, x^{\prime}}^{s+1}}+\left\|w_{1}\right\|_{H_{t, x^{\prime}}^{s}}+\|\widetilde{P}(w)\|_{L_{x_{1}}^{1}\left((-2,2) ; H_{t, x^{\prime}}^{s}\right)}
$$

Here $w_{0}\left(t, x^{\prime}\right)=w\left(t, 0, x^{\prime}\right)$ and $w_{1}\left(t, x^{\prime}\right)=\partial_{x_{1}} w\left(t, 0, x^{\prime}\right)$. In practice, $\widehat{w}\left(\tau, x_{1}, \xi^{\prime}\right)$ will always be localized to a dyadic region in $\left(\tau, \xi^{\prime}\right)$ and hence (4.2) allows us to 
show the inequalities in the hypotheses of Theorem 2.1 .

Now that the parametrix has been constructed we go back to the original roles of $t, x_{1}$ so that $\widetilde{P}$ is an operator that is hyperbolic in $x_{1}$. This also means that the our evolution operators $\mathbf{c}, \mathbf{s}$ are parameterized by $x_{1}$ so that our $w$ takes the form

$$
\begin{aligned}
w(t, x)=\left(\mathbf{c}\left(x_{1}, 0\right) w(\cdot, 0, \cdot)\right)\left(t, x^{\prime}\right)+\left(\mathbf{s}\left(x_{1}, 0\right)\right. & \left.\partial_{x_{1}} w(\cdot, 0, \cdot)\right)\left(t, x^{\prime}\right) \\
& +\int_{0}^{x_{1}}\left(\mathbf{s}\left(x_{1}, y_{1}\right) G\left(\cdot, y_{1}, \cdot\right)\right)\left(t, x^{\prime}\right) d y_{1} .
\end{aligned}
$$

In this section, $\left\{\psi_{\nu}\left(t, x^{\prime}\right)\right\}_{\nu}$ will denote the family of wave packets constructed in section 3.1 except taken as functions of $\left(t, x^{\prime}\right)$ and indexed by $\nu$ rather than $\gamma$. Hence $\nu$ ranges over triples of the form $\nu=\left(k_{\nu},\left(t_{\nu}, x_{\nu}^{\prime}\right), \omega_{\nu}\right)$. We will reserve the notation $\left\{\varphi_{\gamma}(x)\right\}_{\gamma}$ to denote the family of wave packets taken to be functions in $x$.

Let $\widetilde{H}^{ \pm}\left(x, \tau, \xi^{\prime}\right), \widetilde{H}_{k}^{ \pm}\left(x, \tau, \xi^{\prime}\right)$ denote the roots of the principal symbols of $\widetilde{P}, \widetilde{P}^{k}$ respectively. The flow on the cosphere bundle is thus determined by solutions to the ODE

$$
\begin{aligned}
\frac{d t}{d x_{1}} & =-\left(\widetilde{H}_{k}^{+}\right)_{\tau}\left(x_{1}, x^{\prime}, \omega\right) \\
\frac{d x^{\prime}}{d x_{1}} & =-\left(\widetilde{H}_{k}^{+}\right)_{\xi^{\prime}}\left(x_{1}, x^{\prime}, \omega\right) \\
\frac{d \omega}{d x_{1}} & =\left(\widetilde{H}_{k}^{+}\right)_{t, x^{\prime}}\left(x_{1}, x^{\prime}, \omega\right)-\left\langle\omega,\left(\widetilde{H}_{k}^{+}\right)_{t, x^{\prime}}\left(x_{1}, x^{\prime}, \omega\right)\right\rangle \omega \\
\frac{d \Theta}{d x_{1}} & =\Theta\left[\omega \cdot\left(\widetilde{H}_{k}^{+}\right)_{t, x^{\prime}}\left(x_{1}, x^{\prime}, \omega\right)^{T}-\left(\widetilde{H}_{k}^{+}\right)_{t, x^{\prime}}\left(x_{1}, x^{\prime}, \omega\right) \cdot \omega^{T}\right] .
\end{aligned}
$$

with a similar definition of the flow when we consider $\widetilde{H}^{-}$.

Thus given a triple $\nu=\left(k_{\nu},\left(t_{\nu}, x_{\nu}^{\prime}\right), \omega_{\nu}\right)$, set

$$
\psi_{\nu}(t, x)=\psi_{\nu}\left(\Theta_{\nu}\left(x_{1}\right)\left(t-t_{\nu}\left(x_{1}\right), x^{\prime}-x_{\nu}\left(x_{1}\right)^{\prime}\right)+\left(t_{\nu}, x_{\nu}^{\prime}\right)\right)
$$

where $\left.\left(\left(t_{\nu}\left(x_{1}\right), x_{\nu}\left(x_{1}\right)^{\prime}\right), \omega_{\nu}\left(x_{1}\right), \Theta_{\nu}\left(x_{1}\right)\right)\right|_{x_{1}=0}=\left(\left(t_{\nu}, x_{\nu}^{\prime}\right), \omega_{\nu}, I\right)$ and is a solution to (4.3), with $k=k_{\nu}$. These are the traveling wave packets constructed in Section 3 now parameterized by $x_{1}$ rather than $t$.

We first want to show that we need only consider wave packets whose Fourier transforms lie in a small cone about the $\tau$-axis. Let $\Phi, \widetilde{\Phi}$ be smooth functions in $\left(\tau, \xi^{\prime}\right)$ such that $\operatorname{supp}(\Phi) \subset\left\{\left(\tau, \xi^{\prime}\right): \tau \geq 10\left|\xi^{\prime}\right|\right\} \backslash B_{3}(0), \operatorname{supp}(\widetilde{\Phi}) \subset\left\{\left(\tau, \xi^{\prime}\right)\right.$ : $\left.\tau \geq 8\left|\xi^{\prime}\right|\right\} \backslash B_{\frac{1}{2}}(0)$ and identically 1 on the sets $\left\{\left(\tau, \xi^{\prime}\right): \tau \geq 11\left|\xi^{\prime}\right|\right\} \backslash B_{4}(0)$ and $\left\{\left(\tau, \xi^{\prime}\right): \tau \geq 9\left|\xi^{\prime}\right|\right\} \backslash B_{\frac{2}{3}}(0)$ respectively.

For any integral curve $\left(t_{\nu}(z), x_{\nu}(z)^{\prime}, \omega_{\nu}(z)\right)$ of $\widetilde{H}^{ \pm}$on the cosphere bundle, we have that $\left|\omega_{\nu}\left(z_{1}\right)-\omega_{\nu}\left(z_{2}\right)\right| \leq \mathcal{O}(\varepsilon)$ for $z_{1}, z_{2} \in(-2,2)$ as $\left\|a_{i j}-\delta_{i j}\right\|_{L i p},\|\rho-1\|_{L i p}<\varepsilon$ by our assumption in (1.14). Thus by choosing $\varepsilon>0$ to be sufficiently small, we can fix things so that given any wave packet with $\omega_{\nu}\left(z_{0}\right) \notin\left\{\left(\tau, \xi^{\prime}\right): \tau \geq 8\left|\xi^{\prime}\right|\right\}$ for some $z_{0}$ in a finite time interval, then $\omega_{\nu}(z) \notin\left\{\left(\tau, \xi^{\prime}\right): \tau \geq 10\left|\xi^{\prime}\right|\right\}$ for any $z \in(-2,2)$. This implies that $\Phi(D) \tilde{\mathbf{c}}\left(x_{1}, y_{1}\right)=\Phi(D) \tilde{\mathbf{c}}\left(x_{1}, y_{1}\right) \widetilde{\Phi}(D)$ and 
$\Phi(D) \tilde{\mathbf{s}}\left(x_{1}, y_{1}\right)=\Phi(D) \tilde{\mathbf{s}}\left(x_{1}, y_{1}\right) \widetilde{\Phi}(D)$, for any $x_{1}, y_{1} \in(-2,2)$.

Hence in order to get the desired estimates (4.2) for $w$ satisfying (4.1) it suffices to show

$$
\begin{aligned}
&\left\|\Phi \tilde{\mathbf{c}}\left(x_{1}, 0\right) \widetilde{\Phi} h\right\|_{L_{t}^{p} L_{x}^{q}\left([-1,1] \times[-2,2] \times \mathbb{R}_{x^{\prime}}^{n-1}\right)} \leq C\|h\|_{H^{s+1}\left(\mathbb{R}_{t, x^{\prime}}^{n}\right)}, \\
&\left\|\Phi \tilde{\mathbf{s}}\left(x_{1}, y_{1}\right) \widetilde{\Phi} h\right\|_{L_{t}^{p} L_{x}^{q}\left([-1,1] \times[-2,2] \times \mathbb{R}_{x^{\prime}}^{n-1}\right)} \leq C\|h\|_{H^{s}\left(\mathbb{R}_{t, x^{\prime}}^{n}\right)}
\end{aligned}
$$

with $C$ independent of choice of $x_{1}, y_{1} \in[-2,2]$. For simplicity, we will consider the estimates on $\tilde{\mathbf{c}}\left(x_{1}, 0\right)$ as the estimates on $\tilde{\mathbf{s}}\left(x_{1}, y_{1}\right)$ will follow by similar reasoning. Furthermore, we will only consider the half of $\tilde{\mathbf{c}}\left(x_{1}, 0\right)$ that translates packets along the curves of $\widetilde{H}^{+}$as an identical argument establishes dispersive estimates for the other half of the operator.

A crucial step in the proof of the estimates (4.4) is to fix $t$ and realize the family

$$
\left\{\psi_{\nu}(t, \cdot): \omega_{\nu} \in \operatorname{supp}(\widetilde{\Phi}),\left|t_{\nu}\right|<4\right\}
$$

roughly as a frame of functions on $L^{2}\left(\mathbb{R}^{n}\right)$ in the $x$-variable. We will show that this family shares 2 key properties with the wave packet frame $\left\{\varphi_{\gamma}(x)\right\}_{\gamma}$. The first is that a function $\psi_{\nu}(t, \cdot)$ is highly concentrated in space and in frequency much like a single wave packet (though not strictly localized in frequency). To make this rigorous, we will use the weight functions $\mu_{\delta}$ to show that $\psi_{\nu}(t, \cdot)$ is centered at a certain point in $\mathbb{N} \times S^{*}\left(\mathbb{R}_{x}^{n}\right)$ in the sense that its representation in the wave packet frame $\left\{\varphi_{\gamma}(x)\right\}_{\gamma}$ is rapidly decreasing as $\gamma$ moves away from that point. The second property is that these points, the centers of the $\psi_{\nu}(t, \cdot)$, are spread out, similar to the way the collection of indices $\gamma=\left(k_{\gamma}, x_{\gamma}, \omega_{\gamma}\right)$ are evenly spaced. This allows us to prove a key technical lemma analogous to Lemma 2.5 in [6].

Once this is accomplished, we will see that the center of a function $\psi_{\nu}(t, \cdot)$ in the frame $\left\{\varphi_{\gamma}(x)\right\}_{\gamma}$ is the image of the center of the function $\psi_{\nu}(0, \cdot)$ under the transformation at time $t$ determined by a Hamiltonian flow. Specifically, the flow parameterized by $t$ and determined by the Hamiltonian $\left(\tilde{\rho}^{k}\right)^{-\frac{1}{2}}\left\langle\tilde{A}^{k}(x) \xi, \xi\right\rangle^{\frac{1}{2}}$, with $\tilde{A}^{k}(x)$ denoting the matrix of coefficients $\left\{\tilde{a}_{i j}^{k}(x)\right\}_{i j}$ and $k=k_{\nu}$. This entire approach amounts to arguing that the family of $\left\{\psi_{\nu}(t, \cdot)\right\}_{\nu}$ behave roughly as if they were wave packets in $x$ translated along characteristics in $t$. We are then able to adapt the techniques used in Lemmas 6.2 and 6.3 of [6] to complete the proof.

We first reduce the matter to obtaining estimates on wave packets at a fixed frequency shell. Let $S_{k}$ be the set of all $\nu$ such that $\omega_{\nu} \in \operatorname{supp}(\widetilde{\Phi})$ and $k_{\nu}=k$. We consider estimates on the operator $B_{k}$ mapping $l^{2}\left(S_{k}\right)$ to measurable functions on $\mathbb{R} \times[-2,2] \times \mathbb{R}^{n-1}$ given by

$$
B_{k}\left\{c_{\nu}\right\}_{\nu \in S_{k}}=\sum_{\nu \in S_{k}} c_{\nu} \psi_{\nu}(t, x)
$$

We wish to show that there exists $C$ independent of $k$ such that

$$
\left\|B_{k}\left\{c_{\nu}\right\}_{\nu \in S_{k}}\right\|_{L_{t}^{p} L_{x}^{q}\left([-1,1] \times(-2,2) \times \mathbb{R}_{x^{\prime}}^{n-1}\right)} \leq C 2^{k(s+1)}\left\|\left\{c_{\nu}\right\}_{\nu \in S_{k}}\right\|_{l^{2}\left(S_{k}\right)}
$$


for $p, q, s$ as in Theorem 1.1. Thus if $h=\sum_{\nu} c_{\nu} \psi_{\nu}$ for some $h \in L^{2}\left(\mathbb{R}_{t, x}^{n}\right)$ this implies the stronger estimate

$$
\left\|B_{k}\left\{c_{\nu}\right\}_{\nu \in S_{k}}\right\|_{L_{t}^{p} L_{x}^{q}\left([-1,1] \times(-2,2) \times \mathbb{R}_{x^{\prime}}^{n-1}\right)} \leq C 2^{k(s+1)}\left\|h_{k}\right\|_{L^{2}\left(\mathbb{R}_{t, x^{\prime}}^{n}\right)}
$$

where $\left\{h_{k}\right\}_{k \geq 0}$ is an appropriate Littlewood-Paley decomposition of $h$. Since the $B_{k}\left\{c_{\nu}\right\}_{\nu \in S_{k}}$ are dyadically localized to frequencies near $2^{k}$ in the $t, x^{\prime}$ variables, Littlewood-Paley theory will then imply (4.4) as

$$
\begin{aligned}
\left\|\sum_{k} B_{k}\left\{c_{\nu}\right\}_{\nu \in S_{k}}\right\|_{L_{t}^{p} L_{x}^{q}} & \lesssim\left(\sum_{k}\left\|B_{k}\left\{c_{\nu}\right\}_{\nu \in S_{k}}\right\|_{L_{t}^{p} L_{x}^{q}}^{2}\right)^{\frac{1}{2}} \lesssim\left(\sum_{k} 2^{2 k(s+1)}\left\|h_{k}\right\|_{L^{2}\left(\mathbb{R}_{t, x^{\prime}}^{n}\right)}^{2}\right)^{\frac{1}{2}} \\
& \lesssim\|h\|_{H^{s+1}\left(\mathbb{R}_{t, x^{\prime}}^{n}\right)}
\end{aligned}
$$

and $\sum_{k} B_{k}$ represents the half of $\tilde{\mathbf{c}}\left(x_{1}, 0\right)$ that translates packets along $\widetilde{H}^{+}$.

Let $\tilde{\psi} \in C_{0}^{\infty}(\mathbb{R})$ be a smooth bump function equal to 1 on the interval $[-2,2]$ and supported in $\left(-\frac{5}{2}, \frac{5}{2}\right)$. Define $\widetilde{B}_{k}$ as a map from $l^{2}\left(S_{k}\right)$ to measurable functions on $\mathbb{R}^{n+1}$ by

$$
\widetilde{B}_{k}\left\{c_{\nu}\right\}_{\nu \in S_{k}}=\sum_{\nu \in S_{k}} c_{\nu} \tilde{\psi}\left(x_{1}\right) \psi_{\nu}(t, x)
$$

since $\tilde{\psi}$ is compactly supported this is well-defined for any $x_{1} \in \mathbb{R}$. We now focus on proving a stronger inequality than (4.5):

$$
\left\|\widetilde{B}_{k}\left\{c_{\nu}\right\}_{\nu \in S_{k}}\right\|_{L_{t}^{p} L_{x}^{q}\left([-1,1] \times \mathbb{R}^{n}\right)} \leq C 2^{k(s+1)}\left\|\left\{c_{\nu}\right\}_{\nu}\right\|_{l^{2}\left(S_{k}\right)} .
$$

Hence for the rest of this section we will replace $\psi_{\nu}$ by $\tilde{\psi} \psi_{\nu}$ as we will find the property that $\psi_{\nu}$ is defined on all of $\mathbb{R}^{n+1}$ useful in the analysis below.

A duality argument reduces (4.6) to showing

$$
\left\|\widetilde{B}_{k} \widetilde{B}_{k}^{*} G\right\|_{L_{t}^{p} L_{x}^{q}\left([-1,1] \times \mathbb{R}^{n}\right)} \lesssim 2^{2 k(s+1)}\|G\|_{L_{t}^{p^{\prime}} L_{x}^{q^{\prime}}\left([-1,1] \times \mathbb{R}^{n}\right)} .
$$

We now characterize $\widetilde{B}_{k} \widetilde{B}_{k}^{*}$ by

$$
\left(\widetilde{B}_{k} \widetilde{B}_{k}^{*} G\right)(t, x)=\int\left(W_{t, s}^{k} G(s, \cdot)\right)(x) d s
$$

where

$$
\left(W_{t, s}^{k} G(s, \cdot)\right)\left(x_{1}, x^{\prime}\right)=\sum_{\nu} \psi_{\nu}\left(t, x^{\prime}\right) \int_{-1}^{1} \overline{\psi_{\nu}\left(s, y^{\prime}\right)} G(s, \cdot) d y_{1} d y^{\prime} .
$$

The inequality (4.7) will now come from interpolation on the pair of estimates in Theorem 4.1 below (often called the "dispersive estimates") followed by an application of the Hardy-Littlewood-Sobolev inequality. We are now lead to the main result of this section.

Theorem 4.1. The operator $W_{t, s}^{k}$ enjoys the following mapping properties:

$$
\begin{aligned}
& \left\|W_{t, s}^{k} f\right\|_{L^{2}\left(\mathbb{R}^{n}\right)} \leq C\|f\|_{L^{2}\left(\mathbb{R}^{n}\right)} \\
& \left\|W_{t, s}^{k} f\right\|_{L^{\infty}\left(\mathbb{R}^{n}\right)} \leq C 2^{k n}\left(1+2^{k}|t-s|\right)^{-\frac{n-1}{2}}\|f\|_{L^{1}\left(\mathbb{R}^{n}\right)}
\end{aligned}
$$

with $C$ independent of $t, s \in[-1,1]$. 
Begin by setting $K:=\left\{\nu \in S_{k}:\left|t_{\nu}\right|<4\right\}$ and split the integral kernel of $W_{t, s}^{k}$ into 2 parts

$$
\sum_{\nu} \psi_{\nu}(s, y) \psi_{\nu}(t, x)=\sum_{\nu \in K^{C}} \psi_{\nu}(s, y) \psi_{\nu}(t, x)+\sum_{\nu \in K} \psi_{\nu}(s, y) \psi_{\nu}(t, x)
$$

Observe that if $\left|t_{\nu}\right| \geq 4$, and $\varepsilon>0$ is chosen to be sufficiently small in (1.14), then when $x_{1} \in\left(-\frac{5}{2}, \frac{5}{2}\right),\left|t_{\nu}\left(x_{1}\right)\right| \geq\left|t_{\nu}\right|-\left|t_{\nu}\left(x_{1}\right)-t_{\nu}\right| \geq 4-\frac{11}{4}$. Hence $\left|t_{\nu}\left(x_{1}\right)\right|$ stays a uniform distance of $\frac{1}{4}$ away from the interval $[-1,1]$ so by modifying the standard estimates on the localization of $\psi_{\nu}$ in space, we have that

$$
\left|\psi_{\nu}(t, x)\right| \leq C_{N} 2^{\frac{k(n+1)}{4}}\left(1+2^{k}\left|t-t_{\nu}\right|+2^{\frac{k}{2}}\left|x^{\prime}-x_{\nu}^{\prime}\right|\right)^{-N}
$$

for any integer $N$. By choosing $N$ sufficiently large it is not difficult to obtain $C$ independent of $k$ such that

$$
\begin{gathered}
\left|\sum_{\nu \in K^{C}} \psi_{\nu}(s, y) \psi_{\nu}(t, x)\right| \leq C \\
\int_{\mathbb{R}^{n}}\left|\sum_{\nu \in K^{C}} \psi_{\nu}(s, y) \psi_{\nu}(t, x)\right| d x^{\prime} d x_{1} \leq C
\end{gathered}
$$

This shows that the first part of the kernel has the desired mapping properties. It thus suffices to show the estimates assuming that the kernel is sums only over $\nu \in K$.

Since we are looking at wave packets at a fixed frequency $2^{k}$ translated along a fixed flow in what follows we abbreviate $\widetilde{H}_{k}^{+}$as $\widetilde{H}$ and specify whenever we need to work with the rough Hamiltonian. Also, we will always assume implicitly that $\Psi=1$ in the definition of $\widetilde{P}, \widetilde{P}^{k}$ as we are restricting our attention to a sufficiently small cone about the $\tau$-axis.

This first lemma shows that for $\nu \in K, \psi_{\nu}(t, \cdot)$ is indeed concentrated in space and in frequency much like a wave packet.

Lemma 4.2. Suppose $\left(t_{\nu}\left(x_{1}\right), x_{\nu}\left(x_{1}\right)^{\prime}, \omega_{\nu}\left(x_{1}\right)\right)$ is a solution of (4.3) with initial conditions $\left(t_{\nu}, x_{\nu}^{\prime}, \omega_{\nu}\right)$, such that $t_{\nu}(z)=t$ for some $z \in[-16,16]$. Then for any wave packet $\varphi_{\gamma}(x)$ and integer $N$, we have that

$$
\begin{aligned}
& \left|\int \psi_{\nu}(t, x) \varphi_{\gamma}(x) d x\right| \\
& \quad \leq \begin{cases}C_{N} \mu_{N}\left(\gamma ; k_{\nu},\left(z, x_{\nu}(z)^{\prime}\right), \pi\left(\zeta_{\nu}(z), \omega_{\nu}(z)^{\prime}\right)\right) & \left|k_{\nu}-k_{\gamma}\right| \leq 2 \\
C_{N} 2^{-\max \left(k_{\nu}, k_{\gamma}\right) N_{N}} \mu_{N}\left(\gamma ; k_{\nu},\left(z, x_{\nu}(z)^{\prime}\right), \pi\left(\zeta_{\nu}(z), \omega_{\nu}(z)^{\prime}\right)\right) & \left|k_{\nu}-k_{\gamma}\right| \geq 3\end{cases}
\end{aligned}
$$

where $\zeta_{\nu}(z)=\widetilde{H}\left(z, x_{\nu}(z)^{\prime}, \omega_{\nu}(z)\right), \pi(\eta)=\eta /|\eta|$ denotes projection onto the unit sphere, and $C_{N}$ is independent of $\gamma, \nu$.

Therefore, the center of $\psi_{\nu}(t, x)$ in the frame $\left\{\varphi_{\gamma}(x)\right\}_{\gamma}$ is

$$
\left(k_{\nu},\left(z, x_{\nu}(z)^{\prime}\right), \pi\left(\zeta_{\nu}(z), \omega_{\nu}(z)^{\prime}\right)\right) \text {. }
$$

Proof. Throughout this proof $t$ will remain fixed. Let $\eta \mapsto \pi_{\nu}^{\perp}(\eta)$ denote projection onto the subspace orthogonal to $\left(\zeta_{\nu}(z), \omega_{\nu}(z)^{\prime}\right)$. The key idea in this proof is to show the following pair of inequalities:

$$
\left|\widehat{\psi_{\nu}}(t, \eta)\right| \leq C_{N} 2^{-\frac{k(n+1)}{4}}\left(1+2^{-k}\left|\pi_{\nu}^{\perp}(\eta)\right|^{2}+2^{-2 k}|\eta|^{2}\right)^{-N}
$$




$$
\left|\widehat{\psi_{\nu}}(t, \eta)\right| \leq C_{N} 2^{-\frac{3 k(n+1)}{4}} \int_{\operatorname{supp}\left(\widehat{\psi_{\nu}}\right)}\left(1+2^{-k}|\eta-|\left(\tau, \xi^{\prime}\right)\left|\left(\zeta_{\nu}(z), \omega_{\nu}(z)^{\prime}\right)\right|^{2}\right)^{-N} d \tau d \xi^{\prime} .
$$

Indeed, by using the techniques of Lemma 3.2, the lemma follows from these inequalities once it is observed that (4.8) and (4.9) are analogues of (3.19) and (3.21) respectively.

Set $\Phi_{\nu}(t, x)=\Theta_{\nu}\left(x_{1}\right)\left(t-t_{\nu}\left(x_{1}\right), x^{\prime}-x_{\nu}\left(x_{1}\right)^{\prime}\right)$ so that $\psi_{\nu}(t, x)=\psi_{\nu}\left(\Phi_{\nu}(t, x)+\right.$ $\left.\left(t_{\nu}, x_{\nu}^{\prime}\right)\right)$. Since $\Theta_{\nu}$ is orthogonal and $t_{\nu}(z)=t$, standard wave packet estimates give us that

$$
\left|\psi_{\nu}(t, x)\right| \leq C_{N}\left(1+2^{k}\left|\left\langle\omega_{\nu}, \Phi_{\nu}(t, x)\right\rangle\right|+2^{\frac{k}{2}}\left|t_{\nu}(z)-t_{\nu}\left(x_{1}\right)\right|+2^{\frac{k}{2}}\left|x^{\prime}-x_{\nu}\left(x_{1}\right)^{\prime}\right|\right)^{-N}
$$

By performing a Taylor expansion about $z$ on the curve $\left(t_{\nu}\left(x_{1}\right), x_{\nu}\left(x_{1}\right)^{\prime}\right)$ with second order error term $r_{\nu}\left(x_{1}\right)$ we can use the fact that $\Theta_{\nu}\left(x_{1}\right)^{T} \omega_{\nu}=\omega_{\nu}\left(x_{1}\right)$ to write

$$
\begin{aligned}
& \left\langle\Phi_{\nu}(t, x), \omega_{\nu}\right\rangle=\left\langle\left(t-t_{\nu}\left(x_{1}\right), x^{\prime}-x_{\nu}\left(x_{1}\right)^{\prime}\right), \omega_{\nu}\left(x_{1}\right)\right\rangle \\
& \quad=\left\langle\left(t-t_{\nu}(z), x^{\prime}-x_{\nu}(z)^{\prime}\right)-\left(\dot{t}_{\nu}\left(x_{1}\right), \dot{x}_{\nu}\left(x_{1}\right)^{\prime}\right)\left(x_{1}-z\right)+r_{\nu}\left(x_{1}\right), \omega_{\nu}\left(x_{1}\right)\right\rangle .
\end{aligned}
$$

We can now use the fact that $\left(\dot{t}_{\nu}\left(x_{1}\right), \dot{x}_{\nu}\left(x_{1}\right)^{\prime}\right)=-\widetilde{H}_{\tau, \xi^{\prime}}$ and homogeneity to get

$$
\left\langle\Phi_{\nu}(t, x), \omega_{\nu}\right\rangle=\left\langle\left(x_{1}-z, x^{\prime}-x_{\nu}(z)^{\prime}\right),\left(\zeta_{\nu}(z), \omega_{\nu}(z)^{\prime}\right)\right\rangle+e_{\nu}(x)
$$

where $\left|e_{\nu}(x)\right| \lesssim\left|\left(x_{1}-z, x^{\prime}-x_{\nu}(z)^{\prime}\right)\right|^{2}$. By choosing $\varepsilon$ sufficiently small in (1.14), we have $\left|t_{\nu}(z)-t_{\nu}\left(x_{1}\right)\right| \geq \frac{1}{2}\left|z-x_{1}\right|$ implying that

$$
\left|t_{\nu}(z)-t_{\nu}\left(x_{1}\right)\right|+\left|x^{\prime}-x_{\nu}\left(x_{1}\right)^{\prime}\right| \gtrsim\left|\left(x_{1}-z, x^{\prime}-x_{\nu}(z)^{\prime}\right)\right| .
$$

Hence as a result of (4.10) we have that for any $N>0$

$$
\begin{aligned}
& \left|\psi_{\nu}(t, x)\right| \leq \\
& C_{N}\left(1+2^{k}\left|\left\langle\left(x_{1}, x^{\prime}\right)-\left(z, x_{\nu}(z)^{\prime}\right),\left(\zeta_{\nu}(z), \omega_{\nu}(z)^{\prime}\right)\right\rangle\right|+2^{k}\left|\left(x_{1}, x^{\prime}\right)-\left(z, x_{\nu}(z)^{\prime}\right)\right|^{2}\right)^{-N} .
\end{aligned}
$$

In addition, observe that $\partial_{x}^{\alpha} e_{\nu}(x)$ is a finite sum of functions of the form $h_{0}^{k, \alpha}$, $h_{1}^{k, \alpha}$, and $h_{2}^{k, \alpha}$ that have following estimates

$$
\left|h_{l}^{k, \alpha}(x)\right| \leq C_{\alpha} 2^{\frac{k}{2}(|\alpha|+l-2)}\left|\left(x_{1}-z, x^{\prime}-x_{\nu}(z)^{\prime}\right)\right|^{l}
$$

with $C_{\alpha}$ independent of $\nu, k$. We now write the phase of the defining integral

$$
\psi_{\nu}(t, x)=2^{-\frac{k(n+1)}{4}} \int e^{i\left\langle\Phi_{\nu}(t, x),\left(\tau, \xi^{\prime}\right)\right\rangle} h_{k}^{\omega_{\nu}}\left(\tau, \xi^{\prime}\right) d \tau d \xi^{\prime}
$$

as

$$
\begin{aligned}
i\left\langle\Phi_{\nu}(t, x),\left(\tau, \xi^{\prime}\right)\right\rangle=i\left\langle\left( x_{1}-z,\right.\right. & \left.\left.x^{\prime}-x_{\nu}(z)^{\prime}\right),\left(\zeta_{\nu}(z), \omega_{\nu}(z)^{\prime}\right)\right\rangle\left|\left(\tau, \xi^{\prime}\right)\right| \\
& +i e_{\nu}(x)\left|\left(\tau, \xi^{\prime}\right)\right|+i\left\langle\Phi_{\nu}(t, x),\left(\tau, \xi^{\prime}\right)-\left|\left(\tau, \xi^{\prime}\right)\right| \omega_{\nu}\right\rangle .
\end{aligned}
$$

We are now able to integrate by parts in $\left(x_{1}, x^{\prime}\right)$ in the integral defining $\widehat{\psi_{\nu}}(t, \eta)$ as in Lemma 3.2 to obtain (4.8) and (4.9).

To see that the centers of the $\psi_{\nu}(t, \cdot)$ in the frame $\left\{\varphi_{\gamma}\right\}$ are indeed spread out, we show that the pseudodistance between the centers of 2 of these functions at a fixed time slice in the frame $\left\{\varphi_{\gamma}(x)\right\}_{\gamma}$ is bounded below by the pseudodistance between their centers as functions lying on the hyperplane $x_{1}=0$. 
Lemma 4.3. Let

$$
x_{1} \mapsto\left(t_{x_{1}}, x_{x_{1}}^{\prime}, \omega_{x_{1}}\right) \quad \text { and } \quad x_{1} \mapsto\left(s_{x_{1}}, y_{x_{1}}^{\prime}, v_{x_{1}}\right)
$$

denote integral curves of $\widetilde{H}$ projected onto the cosphere with initial values lying in the set

$$
\left\{\left(t, x^{\prime}, \tau, \xi^{\prime}\right) \in(-4,4) \times \mathbb{R}^{n-1} \times \mathbb{R}^{n}: \tau \geq 8\left|\xi^{\prime}\right|\right\} .
$$

Suppose in addition $t=t(z)=s(w)$ for $z, w \in(-16,16)$. Then there exists $a$ uniform constant $c$ such that

$$
\begin{aligned}
d\left(z, x_{z}^{\prime}, \pi\left(\widetilde{H}\left(z, x_{z}^{\prime}, \omega_{z}\right), \omega_{z}^{\prime}\right)\right. & \left.; w, y_{w}^{\prime}, \pi\left(\widetilde{H}\left(w, y_{w}^{\prime}, v_{w}\right), v_{w}^{\prime}\right)\right) \\
& \gtrsim d\left(t_{w}, x_{w}^{\prime}, \omega_{w} ; s_{w}, y_{w}^{\prime}, v_{w}\right) \approx d\left(t_{0}, x_{0}^{\prime}, \omega_{0} ; s_{0}, y_{0}^{\prime}, v_{0}\right)
\end{aligned}
$$

where $\pi$ denotes projection onto the unit sphere as before.

Proof. Consider the map $F:(-16,16) \times \mathbb{R}^{n-1} \times\left\{\left(\tau, \xi^{\prime}\right) \neq 0: \tau \geq 2\left|\xi^{\prime}\right|\right\}$ given by $F\left(z, x^{\prime}, \tau, \xi^{\prime}\right)=\left(z, x^{\prime}, \widetilde{H}\left(z, x^{\prime}, \tau, \xi^{\prime}\right), \xi^{\prime}\right)$ and let $\tilde{D}$ denote the submanifold

$$
(-16,16) \times \mathbb{R}^{n-1} \times\left\{\left(\tau, \xi^{\prime}\right) \in \mathbb{S}^{n-1}: \tau \geq 2\left|\xi^{\prime}\right|\right\}
$$

Since $\widetilde{H}_{\tau}$ is bounded from below on this set, it can be shown that $F$ descends to a map $\widetilde{F}=\left.(\operatorname{Id} \times \pi) \circ F\right|_{\tilde{D}}$ that is a diffeomorphism onto its image with with upper bounds on the pushforward $\left(\widetilde{F}^{-1}\right)_{*}$ under the natural coordinates inheirited by $\mathbb{R}^{2 n}$. This allows us to conclude there exists $c$ uniform such that

$$
\begin{aligned}
|z-w|^{2}+\left|x_{z}^{\prime}-y_{w}^{\prime}\right|^{2}+\left|\pi\left(\widetilde{H}\left(z, x_{z}^{\prime}, \omega_{z}\right), \omega_{z}^{\prime}\right)-\pi\left(\widetilde{H}\left(w, y_{w}^{\prime}, v_{w}\right), v_{w}^{\prime}\right)\right|^{2} \\
\\
\geq c\left(|z-w|^{2}+\left|x_{z}^{\prime}-y_{w}^{\prime}\right|^{2}+\left|\omega_{z}-v_{w}\right|^{2}\right)
\end{aligned}
$$

From here it is not hard to see that the latter quantity is greater than

$$
\tilde{c}\left(|z-w|^{2}+\left|\left(t_{w}, x_{w}^{\prime}\right)-\left(s_{w}, y_{w}^{\prime}\right)\right|^{2}+\left|\omega_{w}-v_{w}\right|^{2}\right)
$$

with uniform constant $\tilde{c}$. We can now apply an argument similar to that used in (4.11) to get

$$
\left\langle\left(\widetilde{H}\left(z, x_{z}^{\prime}, \omega_{z}\right), \omega_{z}^{\prime}\right),\left(w-z, y_{w}^{\prime}-x_{z}^{\prime}\right)\right\rangle=\left\langle\omega_{w},\left(t-t_{w}, y_{w}^{\prime}-x_{w}^{\prime}\right)\right\rangle-e\left(w, y_{w}^{\prime}\right)
$$

with $\left|e\left(w, y_{w}^{\prime}\right)\right| \lesssim\left|\left(t_{z}, x_{z}^{\prime}\right)-\left(s_{w}, y_{w}^{\prime}\right)\right|^{2}$. This now implies that

$$
\begin{aligned}
|z-w|^{2}+\left|x_{z}^{\prime}-y_{w}^{\prime}\right|^{2}+\left|\pi\left(\widetilde{H}\left(z, x_{z}^{\prime}, \omega_{z}\right), \omega_{z}^{\prime}\right)-\pi\left(\widetilde{H}\left(w, y_{w}^{\prime}, v_{w}\right), v_{w}^{\prime}\right)\right|^{2} \\
\quad+\left|\left\langle\left(\widetilde{H}\left(z, x_{z}^{\prime}, \omega_{z}\right), \omega_{z}^{\prime}\right),\left(w-z, y_{w}^{\prime}-x_{z}^{\prime}\right)\right\rangle\right| \\
\gtrsim\left|\omega_{w}-v_{w}\right|^{2}+\left|\left(t_{w}, x_{w}^{\prime}\right)-\left(s_{w}, y_{w}^{\prime}\right)\right|^{2}+\left|\left\langle\omega_{w},\left(t_{w}, x_{w}^{\prime}\right)-\left(s_{w}, y_{w}^{\prime}\right)\right\rangle\right|
\end{aligned}
$$

The first inequality in the conclusion now follows as simple estimates imply that

$$
\left|\left(t_{w}, x_{w}^{\prime}\right)-\left(s_{w}, y_{w}^{\prime}\right)\right| \approx\left|\left(z, x_{z}^{\prime}\right)-\left(w, y_{w}^{\prime}\right)\right| .
$$

The last inequality in the lemma now follows by (3.11).

This result now allows us to prove a key technical lemma that relies on the set of centers being sufficiently sparse. 
Lemma 4.4. For any $\nu \in K=\left\{\nu \in S_{k}:\left|t_{\nu}\right|<4\right\}$, let $S \nu$ denote the index

$$
\left(k,\left(z_{0}, x_{\nu}\left(z_{0}\right)^{\prime}\right), \pi\left(H\left(z_{0}, x_{\nu}\left(z_{0}\right)^{\prime}, \omega_{\nu}\left(z_{0}\right)\right), \omega_{\nu}\left(z_{0}\right)^{\prime}\right)\right)
$$

where $z_{0}$ is such that $t_{\nu}\left(z_{0}\right)=0$. For any $\gamma, \gamma^{\prime}, \gamma_{0}$ we have the following inequalities $\sum_{\nu \in K} \mu_{\delta}(S \nu, \gamma) \leq C_{\delta}\left(1+2^{n\left(k_{\nu}-k_{\gamma}\right)}\right) \quad$ and $\quad \sum_{\nu \in K} \mu_{\delta}\left(\gamma^{\prime}, S \nu\right) \mu_{\delta}\left(S \nu, \gamma_{0}\right) \leq C_{\delta} \mu_{\delta}\left(\gamma^{\prime}, \gamma_{0}\right)$ for some $C_{\delta}$ depending only on $n$ and $\delta>0$.

Proof. The collection $K \cap\left\{\nu: d(S \nu, \gamma) \leq \inf _{\nu} d(S \nu, \gamma)+1\right\}$ is finite, so there exists $\nu_{0}$ such that $d\left(S \nu_{0}, \gamma\right)=\min _{\nu \in K} d(S \nu, \gamma)$. Therefore,

$$
d(S \nu, \gamma) \geq \frac{1}{2} d(S \nu, \gamma)+\frac{1}{12} d\left(S \nu_{0}, S \nu\right)-\frac{1}{2} d\left(S \nu_{0}, \gamma\right) \geq c d\left(\nu_{0}, \nu\right)
$$

The result is now a straightforward adaptation of Lemmas 2.4 and 2.5 in [6].

We are now ready to prove the Theorem:

Proof of Theorem 4.1. Recall that we need only consider the part of the kernel that sums over $\nu \in K$. As such, given an index $\nu$, there always exists $w \in(-16,16)$ such that $t_{\nu}(w)=s$ as it implied by the conditions $\left|t_{\nu}\right| \leq 4$ and $|t| \leq 1$ for $\varepsilon>0$ sufficiently small. Let $\tilde{b}(s, \nu, \gamma)=\int \psi_{\nu}(s, x) \overline{\varphi_{\gamma}}(x) d x$ and let

$$
x_{1} \mapsto\left(t_{\nu}\left(x_{1}\right), x_{\nu}\left(x_{1}\right), \tau_{\nu}\left(x_{1}\right), \xi_{\nu}\left(x_{1}\right)^{\prime}\right)
$$

be the solution to

$$
\begin{aligned}
\frac{d t}{d x_{1}} & =-\widetilde{H}_{\tau}^{+}\left(x, \tau, \xi^{\prime}\right) & \frac{d \tau}{d x_{1}} & =\widetilde{H}_{t}^{+}\left(x, \tau, \xi^{\prime}\right) \\
\frac{d x^{\prime}}{d x_{1}} & =-\widetilde{H}_{\xi}^{+}\left(x, \tau, \xi^{\prime}\right) & \frac{d \xi^{\prime}}{d x_{1}} & =\widetilde{H}_{x^{\prime}}^{+}\left(x, \tau, \xi^{\prime}\right)
\end{aligned}
$$

with initial conditions $\left(t_{\nu}, x_{\nu}^{\prime},\left(\omega_{\nu}\right)_{1}, \omega_{\nu}^{\prime}\right)$. Set

$$
\left(\xi_{\nu}\right)_{1}\left(x_{1}\right)=\widetilde{H}^{+}\left(x_{1}, x_{\nu}\left(x_{1}\right)^{\prime}, \tau_{\nu}\left(x_{1}\right), \xi_{\nu}\left(x_{1}\right)^{\prime}\right)
$$

so that $\left(t_{\nu}\left(x_{1}\right), x_{1}, x_{\nu}\left(x_{1}\right)^{\prime}, \tau_{\nu}\left(x_{1}\right), \xi_{\nu}\left(x_{1}\right)\right)$ is a null bicharacteristic of $\widetilde{P}^{k}$ (with $\left.\xi_{\nu}\left(x_{1}\right)=\left(\left(\xi_{\nu}\right)_{1}\left(x_{1}\right), \xi_{\nu}\left(x_{1}\right)^{\prime}\right)\right)$. It is not difficult to show that for $x_{1} \in(-16,16)$ and $\varepsilon>0$ chosen sufficiently small, this curve admits a reparameterization $t \mapsto$ $\left(t, x_{\nu}(t), \tau_{\nu}(t), \xi_{\nu}(t)\right)$ for $t \in[t(-16), t(16)]$ such that the curve satisfies

$$
\begin{aligned}
& \frac{d x}{d t}=-\nabla_{\xi}\left(\tilde{\rho}^{k}\right)^{-\frac{1}{2}}\left\langle\widetilde{A}^{k}(x) \xi, \xi\right\rangle^{\frac{1}{2}}=\left(H_{k}\right)_{\xi}(x, \tau, \xi), \\
& \frac{d \xi}{d t}=\nabla_{x}\left(\tilde{\rho}^{k}\right)^{-\frac{1}{2}}\left\langle\widetilde{A}^{k}(x) \xi, \xi\right\rangle^{\frac{1}{2}}=-\left(H_{k}\right)_{x}(x, \tau, \xi)
\end{aligned}
$$

where $H_{k}(x, \tau, \xi)=\tau-\left(\tilde{\rho}^{k}\right)^{-\frac{1}{2}}\left\langle\widetilde{A}^{k}(x) \xi, \xi\right\rangle^{\frac{1}{2}}$.

By homogeneity of $\widetilde{H}$ and the fact that $\left(\left(\omega_{\nu}\right)_{1}\left(x_{1}\right), \omega_{\nu}\left(x_{1}\right)^{\prime}\right)=\pi\left(\tau_{\nu}\left(x_{1}\right), \xi_{\nu}\left(x_{1}\right)^{\prime}\right)$, we have

$$
\pi\left(\widetilde{H}\left(z, x_{\nu}(z)^{\prime}, \omega_{\nu}(z)\right), \omega_{\nu}(z)^{\prime}\right)=\pi\left(\xi_{\nu}(z)\right)
$$

for any $z$. Now let $\chi_{t}$ denote the transformation on $S^{*}\left(\mathbb{R}_{x}^{n}\right)=\mathbb{R}_{x}^{n} \times \mathbb{S}^{n-1}$ induced by projecting the flow (4.13) down to the cosphere bundle. We now have that if $w$ is such that $t_{\nu}(w)=s$, then

$$
\left(k_{\nu},\left(w, x(w)^{\prime}\right), \pi(\xi(w))\right)=\chi_{s}(S \nu) .
$$


This, in conjunction with Lemma 4.2, yields

$$
|\tilde{b}(s, \nu, \gamma)| \leq \begin{cases}C_{N} \mu_{N}\left(\gamma, \chi_{s}(S \nu)\right) & \left|k_{\nu}-k_{\gamma}\right| \leq 2 \\ C_{N} 2^{-\max \left(k_{\nu}, k_{\gamma}\right) N} \mu_{N}\left(\gamma, \chi_{s}(S \nu)\right) & \left|k_{\nu}-k_{\gamma}\right| \geq 3\end{cases}
$$

Hence the $\psi_{\nu}(t, x)$ do indeed behave roughly as if they were wave packets originating on the $t=0$ hyperplane and translated along characteristics parameterized by $t$.

We thus have

$$
\left(W_{t, s}^{k} \varphi_{\gamma}\right)(x)=\sum_{\nu} \psi_{\nu}(t, x) \int \psi_{\nu}(s, y) \overline{\varphi_{\gamma}(y)} d y=\sum_{\nu} \psi_{\nu}(t, x) \tilde{b}(s, \nu, \gamma)
$$

Hence, the matrix of the operator $W_{t, s}^{k}$ satisfies

$$
\begin{aligned}
& \left|\int \overline{\varphi_{\gamma^{\prime}}}\left(W_{t, s}^{k}\right) \varphi_{\gamma}(t, x) d x\right| \leq \sum_{\nu}\left|\tilde{b}(s, \nu, \gamma) \tilde{b}\left(t, \nu, \gamma^{\prime}\right)\right| \\
& \quad \leq C_{N} \sum_{\nu} \mu_{N}\left(S \nu, \chi_{-t}(\gamma)\right) \mu_{N}\left(S \nu, \chi_{-s}\left(\gamma^{\prime}\right)\right) \leq \tilde{C}_{N} \mu_{N}\left(\gamma, \chi_{t-s}\left(\gamma^{\prime}\right)\right)
\end{aligned}
$$

Thus $W_{t, s}^{k}$ is an operator with matrix in $\mathcal{M}^{0}\left(\chi_{t-s}\right)$ and the constant appearing in (3.8) can be taken to be independent of $k, t, s$. Hence $W_{t} W_{s}^{*}$ has the desired $L^{2}$ mapping properties.

To show the second estimate, we wish to show the following bound on the kernel of $W_{t} W_{s}^{*}$

$$
\left|\sum_{\nu \in K} \psi_{\nu}(t, x) \psi_{\nu}(s, y)\right| \leq C 2^{k n}\left(1+2^{k}|t-s|\right)^{-\frac{n-1}{2}}
$$

with $C$ independent of $x, y$. Begin by observing the upper bound

$$
\left|\sum_{\nu \in K} \psi_{\nu}(t, x) \psi_{\nu}(s, y)\right| \leq \sum_{\nu \in K} \sum_{\gamma, \gamma^{\prime}}\left|\varphi_{\gamma}(x) \varphi_{\gamma^{\prime}}(y)\right||\tilde{b}(t, \nu, \gamma)|\left|\tilde{b}\left(s, \nu, \gamma^{\prime}\right)\right|
$$

When $\left|k_{\gamma}-k_{\nu}\right| \geq 3$, we can use the extra power of $2^{-\max \left(k_{\gamma}, k_{\nu}\right) N}$ to get the upper bound

$$
\sum_{\nu \in K}\left(\sum_{\left|k_{\gamma}-k_{\nu}\right| \geq 3} \sum_{\gamma^{\prime}}+\sum_{\left|k_{\gamma^{\prime}}-k_{\nu}\right| \geq 3} \sum_{\left|k_{\gamma}-k_{\nu}\right| \leq 2}\right)\left|\varphi_{\gamma}(x) \varphi_{\gamma^{\prime}}(y)\right||\tilde{b}(t, \nu, \gamma)|\left|\tilde{b}\left(s, \nu, \gamma^{\prime}\right)\right| \leq C .
$$

It now suffices to restrict our attention to the sum

$$
\left|\sum_{\nu \in K} \psi_{\nu}(t, x) \psi_{\nu}(s, y)\right| \leq \sum_{\nu \in K} \sum_{\left|k_{\gamma}-k_{\nu}\right|,\left|k_{\gamma^{\prime}}-k_{\nu}\right| \leq 2}\left|\varphi_{\gamma}(x) \varphi_{\gamma^{\prime}}(y)\right||\tilde{b}(t, \nu, \gamma)|\left|\tilde{b}\left(s, \nu, \gamma^{\prime}\right)\right| .
$$

Using Lemma 4.4, this sum is dominated by

$$
C_{N} \sum_{\left|k_{\gamma}-k_{\nu}\right| \leq 2} \sum_{\left|k_{\gamma^{\prime}}-k_{\nu}\right| \leq 2}\left|\varphi_{\gamma}(x) \varphi_{\gamma^{\prime}}(y)\right| \mu_{N}\left(\gamma, \chi_{t-s}\left(\gamma^{\prime}\right)\right)
$$


By the arguments in Lemma 6.2 of [6], it suffices to show Lemma 6.3 of [6] holds under the alternate assumption that the Hamiltonian $H(x, \xi)$ satisfies

$$
\begin{array}{r}
\sum_{|\alpha|+|\beta| \leq 2,|\alpha| \leq 1} \sup _{\frac{1}{2} \leq|\xi| \leq 2}\left|\partial_{x}^{\alpha} \partial_{\xi}^{\beta}(H(x, \xi)-|\xi|)\right|+\sum_{|\alpha|=2} \int_{-16}^{16} \sup _{\frac{1}{2} \leq|\xi| \leq 2}\left|\partial_{x}^{\alpha} H\left(x_{1}, \cdot, \cdot\right)\right| d x_{1} \\
\leq \tilde{\varepsilon}
\end{array}
$$

for some sufficiently small but fixed $\tilde{\varepsilon}>0$ and the slightly weaker conclusion that

$$
\int_{\Omega}(1+\lambda|\langle\tilde{\omega}(\omega), y-\tilde{x}(\omega)\rangle|)^{-2} d \omega \leq C \lambda^{-\frac{n-1}{2}}
$$

where $\Omega:=\left\{\left(\omega_{1}, \omega^{\prime}\right) \in \mathbb{S}^{n-1} \subset \mathbb{R}^{n}: \omega_{1} \geq 8\left|\omega^{\prime}\right|\right\}$. Indeed, (4.14) can be arranged by scaling the coefficients by a factor of $R$ as

$$
\int_{-2}^{2} \sup _{\left|x^{\prime}\right| \leq 2} \sum_{i, j}\left|\partial_{x}^{\beta}\left(a_{i j}\left(R\left(x_{1}, x^{\prime}\right)\right)-\delta_{i j}\right)\right|+\left|\partial_{x}^{\beta}\left(\rho\left(R\left(x_{1}, x^{\prime}\right)\right)-1\right)\right| d x_{1} \leq C R^{1-\frac{1}{r}} .
$$

Consider the Hamiltonian flow for $\eta$ such that $\frac{\eta}{|\eta|} \in \Omega$

$$
\left\{\begin{array}{l}
\frac{d x}{d s}(s, \eta)=\nabla_{\xi} H(x(s, \eta), \xi(s, \eta)) \\
\frac{d \xi}{d s}(s, \eta)=-\nabla_{x} H(x(s, \eta), \xi(s, \eta)) \\
x(0, \eta)=0, \quad \xi(0, \eta)=\eta
\end{array}\right.
$$

When $\frac{1}{2} \leq|\eta| \leq 2$, and $|s| \leq 1$ we have

$$
|\xi(s, \eta)-\eta| \leq \mathcal{O}(\tilde{\varepsilon}) \quad\left|x(s, \eta)-s \frac{\eta}{|\eta|}\right| \leq \mathcal{O}(\tilde{\varepsilon})
$$

as the map $\eta \mapsto \frac{\eta}{|\eta|}$ has bounded derivatives on this set. Differentiation with respect to $\eta$ in the equations above now allows us to conclude that

$$
\begin{aligned}
& \left|\frac{\partial x}{\partial \eta}(1, \eta)-\left(\frac{\mathbf{I}}{|\eta|}-\frac{\eta \cdot \eta^{T}}{|\eta|^{3}}\right)\right|+\left|\frac{\partial \xi}{\partial \eta}(1, \eta)-\mathbf{I}\right| \\
& \quad \leq \mathcal{O}(\tilde{\varepsilon})+\int_{0}^{1} C\left(\| \partial_{x}^{2}\left(A\left(x_{1}(s), \cdot\right) \|_{L^{\infty}}+1\right)\left|\frac{\partial x}{\partial \eta}(s, \eta)-\left(\frac{\mathbf{I}}{|\eta|}-\frac{\eta \cdot \eta^{T}}{|\eta|^{3}}\right)\right| d s\right.
\end{aligned}
$$

with $\left\|\partial_{x}^{2} A\left(x_{1}, \cdot\right)\right\|_{L_{x^{\prime}}^{\infty}}=\sum_{|\alpha|=2} \sum_{i j}\left\|\partial_{x}^{\alpha} a_{i j}\left(x_{1}, \cdot\right)\right\|_{L_{x^{\prime}}^{\infty}}$. Since $d x_{1} / d s$ can be uniformly bounded from below, Gronwall's inequality now gives us that

$$
\left|\frac{\partial x}{\partial \eta}(1, \eta)-\left(\frac{\mathbf{I}}{|\eta|}-\frac{\eta \cdot \eta}{|\eta|^{3}}\right)\right|+\left|\frac{\partial \xi}{\partial \eta}(1, \eta)-\mathbf{I}\right| \leq \mathcal{O}(\tilde{\varepsilon})
$$

The rest of arguments in Lemma 6.3 now imply the desired conclusion under this weaker assumption.

We are now able to prove Theorem 1.1.

Proof of Theorem 1.1. We now show that the hypotheses of Theorem 2.1 are satisfied with $\sigma=0$. To do this, we will actually apply the parametrix and the estimates of this section to the pseudodifferential operator $\widetilde{P}^{k}$, the operator defined in Section 3 with coefficients truncated to frequency $2^{\frac{k}{2}}$. 
Let $w(t, x)$ denote the function produced by Theorem 3.7 as a solution to the Cauchy problem (3.27) with initial data $\psi \Gamma_{k} u(\cdot, 0, \cdot), \partial_{1} \psi \Gamma_{k} u(\cdot, 0, \cdot)$ and driving force $\widetilde{P}^{k}\left(\psi \Gamma_{k} u\right)$ (recall that $\psi\left(x_{1}\right)$ is the smooth cutoff such that $\left.\psi\right|_{\left[-\frac{3}{2}, \frac{3}{2}\right]} \equiv 1$ defined in 2.1). By the observations at the beginning of this section concerning $\Phi(D), \widetilde{\Phi}(D)$ and the fact that the Cauchy data determining $w$ all have partial Fourier transforms in $t, x^{\prime}$ localized to a sufficiently small cone about the $\tau$-axis, we can conclude that $\operatorname{supp}(\widehat{w})\left(\cdot, x_{1}, \cdot\right) \subset\left\{\left(\tau, \xi^{\prime}\right): \tau \geq 6\left|\xi^{\prime}\right|\right\}$. Hence an easy application of the flux estimates of Section 2.2 shows uniqueness of solutions to the PDE with the given inital data and hence $w=\psi \Gamma_{k} u$. Therefore, the dispersive estimates of this section and the fact that $P^{k}\left(\psi \Gamma_{k} u\right)$ is localized to frequency $2^{k}$ in $t, x^{\prime}$ imply that

$$
\begin{aligned}
& \left\|\psi \Gamma_{k} u\right\|_{L_{t}^{p} L_{x}^{q}\left([-1,1] \times(-2,2) \times \mathbb{R}^{n-1}\right)} \\
& \lesssim 2^{k s}\left(2^{k}\left\|\psi \Gamma_{k} u(\cdot, 0, \cdot)\right\|_{H_{t, x^{\prime}}^{s+1}}+\left\|\partial_{x_{1}} \psi \Gamma_{k} u(\cdot, 0, \cdot)\right\|_{H_{t, x^{\prime}}^{s}}+\left\|\widetilde{P}^{k}\left(\psi \Gamma_{k} u\right)\right\|_{L_{x_{1}}^{1} L_{t, x^{\prime}}\left(\mathbb{R}^{n}\right)}\right) .
\end{aligned}
$$

The estimate of Theorem 2.1 now follows by observing the following inequality

$$
\begin{aligned}
\left\|\widetilde{P}^{k}\left(\psi \Gamma_{k} u\right)\right\|_{L_{x_{1}}^{1} L_{t, x^{\prime}}^{2}} & \lesssim\left\|\left(\widetilde{P}^{k}-\widetilde{P}\right)\left(\psi \Gamma_{k} u\right)\right\|_{L_{x_{1}}^{1} L_{t, x^{\prime}}^{2}}+\left\|\widetilde{P}\left(\psi \Gamma_{k} u\right)\right\|_{L_{x_{1}}^{1} L_{t, x^{\prime}}^{2}} \\
& \lesssim\left\|\nabla_{t, x}\left(\psi \Gamma_{k} u\right)\right\|_{L_{x_{1}}^{\infty} L_{t, x^{\prime}}^{2}}^{2}+\left\|P\left(\psi \Gamma_{k} u\right)\right\|_{L_{x_{1}}^{1} L_{t, x^{\prime}}^{2}}^{2}
\end{aligned}
$$

We now conclude this section with an important corollary:

Corollary 4.5. Suppose $w \in C^{\infty}\left(\mathbb{R}^{n+1}\right)$ satisfies

$$
\operatorname{supp}\left(\widehat{w}\left(\cdot, x_{1}, \cdot\right)\right) \subset\left\{\left(\tau, \xi^{\prime}\right): \tau \geq 12\left|\xi^{\prime}\right|\right\} \backslash B_{\frac{3}{4}}(0) \cap\left\{\left(\tau, \xi^{\prime}\right): \frac{1}{64} \lambda \leq\left|\left(\tau, \xi^{\prime}\right)\right| \leq 64 \lambda\right\} .
$$

for all $x_{1} \in \mathbb{R}$. Assume also $\widetilde{P}^{\lambda}$ is the operator obtained by regularizing the coefficients of $\widetilde{P}$ and truncating them to frequencies less than $2 \lambda^{1-\delta}$, for some $\delta>0$. Then for $\lambda$ sufficiently large, there exists $C_{\delta}$ independent of $\lambda, w$ such that

$$
\begin{gathered}
\|w\|_{L_{t}^{p} L_{x}^{q}\left(\mathbb{R} \times(-2,2) \times \mathbb{R}^{n-1}\right)} \leq C_{\delta} \lambda^{s}\left(\|w\|_{L_{x_{1}}^{\infty}\left((-2,2) ; H_{t, x^{\prime}}^{1}\right)}+\left\|\partial_{1} w\right\|_{L_{x_{1}}^{\infty}\left((-2,2) ; L_{t, x^{\prime}}^{2}\right)}\right. \\
\left.+\left\|\widetilde{P}^{\lambda} w\right\|_{L_{x_{1}}^{1} L_{t, x^{\prime}}^{2}}^{2}\left((-2,2) \times \mathbb{R}^{n}\right)\right),
\end{gathered}
$$

with $s, p, q$ as in Theorem 1.1.

Proof. Let $\left\{\phi_{l}(t)\right\}_{l \in \mathbb{Z}}$ be a smooth partition of unity on $\mathbb{R}$ such that $\phi_{l}(t)$ is supported in $(l-1, l+1)$ and $\phi_{l}(t)=\phi_{0}(t-l)$. A straightforward argument using Lemma 2.4 and Minkowski's inequality shows that

$$
\|w\|_{L_{t}^{p} L_{x}^{q}\left(\mathbb{R} \times(-2,2) \times \mathbb{R}^{n-1}\right)} \lesssim\left(\sum_{l}\left\|\phi_{l} w\right\|_{L_{t}^{p} L_{x}^{q}\left(\mathbb{R} \times(-2,2) \times \mathbb{R}^{n-1}\right)}^{2}\right)^{\frac{1}{2}} .
$$

Let $\widetilde{\Omega}\left(\tau, \xi^{\prime}\right)$ be a smooth function on $\mathbb{R}^{n}$ supported in $\left\{\left(\tau, \xi^{\prime}\right): \tau>11\left|\xi^{\prime}\right|\right\} \backslash B_{\frac{1}{2}}(0)$ and identically 1 on the set $\left\{\left(\tau, \xi^{\prime}\right): \tau \geq 12\left|\xi^{\prime}\right|\right\} \backslash B_{\frac{3}{4}}(0)$. Also, let $\tilde{\theta}\left(\tau, \xi^{\prime}\right)$ be a smooth function supported in $\left\{\left(\tau, \xi^{\prime}\right): \frac{1}{65} \leq\left|\left(\tau, \xi^{\prime}\right)\right| \leq 65\right\}$ and identically one on the set $\left\{\left(\tau, \xi^{\prime}\right): \frac{1}{64} \leq\left|\left(\tau, \xi^{\prime}\right)\right| \leq 64\right\}$. Now define $\widetilde{\Omega}_{\lambda}(\tau, \xi)=\widetilde{\Omega}\left(\tau, \xi^{\prime}\right) \tilde{\theta}\left(\lambda^{-1}\left(\tau, \xi^{\prime}\right)\right)$. Since $\left(I-\widetilde{\Omega}_{\lambda}(D)\right) w=0$, we now have that

$$
\begin{aligned}
\left\|\phi_{l} w\right\|_{L_{t}^{p} L_{x}^{q}\left(\mathbb{R} \times(-2,2) \times \mathbb{R}^{n-1}\right)} & \leq\left\|\widetilde{\Omega}_{\lambda} \phi_{l} w\right\|_{L_{t}^{p} L_{x}^{q}\left([-l-1,-l+1] \times(-2,2) \times \mathbb{R}^{n-1}\right)} \\
& +\left\|\left[I-\widetilde{\Omega}_{\lambda}, \phi_{l}\right] w\right\|_{L_{t}^{p} L_{x}^{q}\left([-l-1,-l+1] \times(-2,2) \times \mathbb{R}^{n-1}\right)}
\end{aligned}
$$


By applying flux estimates as in the proof of Theorem 1.1 it can be shown that when $\lambda$ is sufficiently large, $\widetilde{\Omega}_{\lambda} \phi_{l} w$ is indeed represented by the parametrix of Section 3 as a solution to an equation involving $\widetilde{P}^{\lambda}$, yielding the inequality

$$
\begin{aligned}
& \left\|\widetilde{\Omega}_{\lambda}(D) \phi_{l} w\right\|_{L_{t}^{p} L_{x}^{q}\left([-l-1,-l+1] \times(-2,2) \times \mathbb{R}^{n-1}\right)} \\
& \leq C_{\delta} \lambda^{s}\left(\left\|\widetilde{\Omega}_{\lambda} \phi_{l} w(\cdot, 0, \cdot)\right\|_{H_{t, x^{\prime}}^{1}}+\left\|\widetilde{\Omega}_{\lambda} \phi_{l} \partial_{1} w(\cdot, 0, \cdot)\right\|_{L_{t, x^{\prime}}^{2}}+\left\|\phi_{l} \widetilde{P}^{\lambda} w\right\|_{L_{x_{1}}^{1} L_{t, x^{\prime}}}^{2}\left((-2,2) \times \mathbb{R}^{n}\right)\right. \\
& \left.\quad+\left\|\left[\widetilde{P}^{\lambda}, \widetilde{\Omega}_{\lambda} \phi_{l}\right] w\right\|_{L_{x_{1}}^{1} L_{t, x^{\prime}}}^{2}\left((-2,2) \times \mathbb{R}^{n}\right)\right)
\end{aligned}
$$

Consider the sum over $l$ of the squares of the first line on the right hand side of the inequality. It is straightforward to see that this sum is dominated by

$$
\|w\|_{L_{x_{1}}^{\infty}\left((-2,2) ; H_{t, x^{\prime}}^{1}\right)}+\left\|\partial_{1} w\right\|_{L_{x_{1}}^{\infty}\left((-2,2) ; L_{t, x^{\prime}}^{2}\right)}+\left\|\widetilde{P}^{\lambda} w\right\|_{L_{x_{1}}^{1} L_{t, x^{\prime}}^{2}\left((-2,2) \times \mathbb{R}^{n}\right)} .
$$

It now suffices to consider the commutator terms. Let $R_{\theta}=\sum_{l} e^{i \theta l} \widetilde{\Omega}_{\lambda} \phi_{l}$ so that $R_{\theta}$ is a pseudodifferential operator in $t, x^{\prime}$ with uniform symbol estimates in $S_{1,0}^{0}$ over $\theta$. Since $\left[\widetilde{P}^{\lambda}, R_{\theta}\right]$ involves partial derivatives in $x_{1}$ of order at most 1 , we can easily adapt the proof of Lemma 2.3 to get that

$$
\left\|\left[\widetilde{P}^{\lambda}, R_{\theta}\right] w\left(x_{1}, \cdot\right)\right\|_{L_{t, x^{\prime}}^{2}} \lesssim\left\|w\left(x_{1}, \cdot\right)\right\|_{H_{t, x^{\prime}}^{1}}+\left\|\partial_{1} w\left(x_{1}, \cdot\right)\right\|_{L_{t, x^{\prime}}^{2}} .
$$

Applying Lemma 2.4 and Minkowski's inequality now yields

$\left.\sum_{l}\left\|\left[\widetilde{P}^{\lambda}, \widetilde{\Omega}_{\lambda} \phi_{l}\right] w\right\|_{L_{x_{1}}^{1} L_{t, x^{\prime}}^{2}\left((-2,2) \times \mathbb{R}^{n}\right)}^{2} \lesssim\|w\|_{L_{x_{1}}^{\infty}\left((-2,2) ; H_{t, x^{\prime}}^{1}\right)}^{2}+\left\|\partial_{1} w\right\|_{L_{x_{1}}^{\infty}\left((-2,2) ; L_{t, x^{\prime}}\right)}^{2}\right)^{2}$

By similar considerations

$$
\begin{aligned}
\sum_{l}\left\|\left[I-\widetilde{\Omega}_{\lambda}, \phi_{l}\right] w\right\|_{L_{t}^{p} L_{x}^{q}\left([-l-1,-l+1] \times(-2,2) \times \mathbb{R}^{n-1}\right)}^{2} & \\
& \lesssim\|w\|_{L_{x_{1}}^{\infty}\left((-2,2) ; H_{t, x^{\prime}}^{1}\right)}^{2}+\left\|\partial_{1} w\right\|_{L_{x_{1}}^{\infty}\left((-2,2) ; L_{t, x^{\prime}}^{\prime}\right.}^{2} .
\end{aligned}
$$

\section{Estimates VIA SCALING}

In this section we show the truncation/rescaling arguments needed to prove Theorem 1.2. Begin by smoothing the coefficients of the operator $\widetilde{P}$ as constructed in Section 3. Let $\left\{\beta_{k}\right\}_{k}$ be a Littlewood Paley decomposition in $x$ as before. Set $\varsigma=\frac{2}{3+\alpha}$ with $\alpha$ as in Theorem 1.2 and recall that $\sigma=\frac{1-\alpha}{3+\alpha}$. For each $\tilde{a}_{i j}=a_{i j} / a_{11}$, $\tilde{\rho}=\rho / a_{11}$ define

$$
\tilde{a}_{i j}^{k}=\sum_{l<k \varsigma} \beta_{l}(D) \tilde{a}_{i j} \quad \tilde{\rho}^{k}=\sum_{l<k \varsigma} \beta_{l}(D) \tilde{\rho} .
$$

Let $a$ denote an arbitrary function of the form $\tilde{a}_{i j}-\delta_{i j}$ or $\tilde{\rho}-1$ and $a^{k}$ its regularized counterpart $\tilde{a}_{i j}^{k}$ or $\tilde{\rho}-1$. Observe that we can write $\beta_{l}(D) a=\beta_{l}(D)\langle D\rangle^{-\kappa}\left(\langle D\rangle^{\kappa} a\right)$. Set $\tilde{\phi}_{l}=\mathcal{F}^{-1}\left\{\beta_{l}(\xi)\langle\xi\rangle^{-\kappa}\right\}$ so that $\beta_{l}(D) a=\left(\langle D\rangle^{\kappa} a\right) * \tilde{\phi}_{l}$. Using integration by parts we have the following estimates on $\tilde{\phi}_{l}$

$$
\left|y^{\beta_{1}} \partial_{y}^{\beta_{2}} \tilde{\phi}_{l}(y)\right| \leq C_{\beta_{1}, \beta_{2}} 2^{l\left(n-\left|\beta_{1}\right|+\left|\beta_{2}\right|-\kappa\right)}
$$


This yields the following estimates on the $\beta_{l}(D) a$

$$
\begin{aligned}
& \left|\partial_{x}^{\beta}\left(\beta_{l}(D) a\right)(x)\right|=\left|\left(\langle D\rangle^{\kappa} a\right) *\left(\partial_{x}^{\beta} \tilde{\phi}_{l}\right)(x)\right| \\
& \leq C_{\beta, n} 2^{l(n-1+|\beta|-\kappa) .} \\
& \quad \int\left\|\left(\langle D\rangle^{\kappa} a\right)\left(x_{1}-y_{1}, \cdot\right)\right\|_{L_{x^{\prime}}^{r}\left(\mathbb{R}^{n-1}\right)}\left(1+\left|2^{l} y_{1}\right|^{2}\right)^{-1} 2^{l} d y_{1}\left\|\left(1+\left|2^{l} y^{\prime}\right|^{2}\right)^{-n}\right\|_{L^{r^{\prime}}} \\
& \quad \leq C_{\beta, n} 2^{l(|\beta|-1-\alpha)} M\left(\left\|\left(\langle D\rangle^{\kappa} a\right)\left(w_{1}, \cdot\right)\right\|_{L_{x^{\prime}}^{r}}\right)\left(x_{1}\right)
\end{aligned}
$$

Here $M\left(\left\|\left(\langle D\rangle^{\kappa} a\right)\left(w_{1}, \cdot\right)\right\|_{L^{r}}\right)\left(x_{1}\right)$ denotes the maximal function associated to the $L^{r}(\mathbb{R})$ function $w_{1} \mapsto\left\|\left(\langle D\rangle^{\kappa} a\right)\left(w_{1}, \cdot\right)\right\|_{L^{r}}$. The last inequality follows by the result on maximal functions cited in section 3. Let $\varrho$ be the $L^{r}(\mathbb{R})$ function given by

$\varrho\left(x_{1}\right)=\sum_{i j} M\left(\left\|\langle D\rangle^{\kappa}\left(\tilde{a}_{i j}-\delta_{i j}\right)\left(w_{1}, \cdot\right)\right\|_{L_{x^{\prime}}^{r}}\right)\left(x_{1}\right)+M\left(\left\|\langle D\rangle^{\kappa}(\tilde{\rho}-1)\left(w_{1}, \cdot\right)\right\|_{L_{x^{\prime}}^{r}}\right)\left(x_{1}\right)$.

Note that this definition of $\varrho$ is different from the one used in section 3 . This now yields the following inequalities for $a$ in the form above

$$
\begin{gathered}
\left|a(x)-a^{k}(x)\right| \leq \sum_{l \geq k \varsigma}\left|\beta_{l}(D) a(x)\right| \leq C \varrho\left(x_{1}\right) \sum_{l \geq k \varsigma} 2^{-l(1+\alpha)} \leq C^{\prime} 2^{-k \varsigma(1+\alpha)} \varrho\left(x_{1}\right), \\
\left|\partial_{x}^{\beta} a^{k}(x)\right| \leq \sum_{l<k \varsigma}\left|\partial_{k}^{\beta} \beta_{l}(D) a(x)\right| \leq C_{\beta} 2^{k \varsigma(|\beta|-1-\alpha)} \varrho\left(x_{1}\right) \quad \text { for }|\beta| \geq 2
\end{gathered}
$$

In addition, the $C^{1}$ norm of $\tilde{a}_{i j}^{k}, \tilde{\rho}^{k}$ is uniformly bounded as $\tilde{a}_{i j}, \tilde{\rho}$ are Lipschitz functions.

Let $\left\{I_{m}^{k}\right\}$ be a collection of disjoint maximal subintervals of $(-2,2)$ indexed by $m$ ranging over positive integers such that $\cup_{m} I_{m}^{k}=(-2,2),\left|I_{m}^{k}\right| \leq 2^{-k \sigma}$, and $\int_{I_{m}^{k}} \varrho\left(x_{1}\right) d x_{1} \leq 2^{-k \sigma}$. Let $K_{1}$ be the cardinality of the set $\left\{I_{m}^{k}: \int_{I_{m}^{k}} \varrho\left(x_{1}\right) d x_{1}=\right.$ $\left.2^{-k \sigma}\right\}$ and $K_{2}$ the cardinality of the set $\left\{I_{m}^{k}:\left|I_{m}^{k}\right|=2^{-k \sigma}\right\}$. By maximality of the subintervals, $K_{1}+K_{2}$ is at least the cardinality of the collection $\left\{I_{m}^{k}\right\}$. Observe that

$$
2^{-k \sigma} K_{1} \leq \int_{-2}^{2} \varrho\left(x_{1}\right) d x_{1} \leq C \quad 2^{-k \sigma} K_{2} \leq 4
$$

and hence there is at most $\mathcal{O}\left(2^{k \sigma}\right)$ subintervals in the collection. Now let $\chi_{m}^{k}\left(x_{1}\right)$ be the characteristic function of the interval $I_{m}^{k}$. This yields the following estimate

$$
\begin{aligned}
\int \sup _{x^{\prime}}\left(\chi_{m}^{k}\left(x_{1}\right)\left|\tilde{a}_{i j}^{k}(x)-\tilde{a}_{i j}(x)\right|\right) d x_{1} & \leq C \int \chi_{m}^{k}\left(x_{1}\right) 2^{-\varsigma(1+\alpha)} \varrho\left(x_{1}\right) d x_{1} \\
& \leq C 2^{(-\sigma-\varsigma(1+\alpha)) k}=C 2^{-k}
\end{aligned}
$$

as a simple computation shows that $\sigma+\varsigma(1+\alpha)=1$. An analogous inequality holds for $\tilde{\rho}$. Next observe that for any multi-index $\beta$ such that $|\beta|=2$

$$
\begin{aligned}
\int \chi_{m}^{k}\left(2^{-k \sigma} x_{1}\right) \sup _{x^{\prime}} & \left|\partial_{x}^{\beta}\left(\tilde{a}_{i j}^{k}\left(2^{-k \sigma} x\right)-\delta_{i j}\right)\right| d x_{1} \\
& \leq C 2^{-2 k \sigma+k \varsigma(1-\alpha)} \int \chi_{m}^{k}\left(2^{-k \sigma} x_{1}\right) \varrho\left(2^{-k \sigma} x_{1}\right) d x_{1} \leq C .
\end{aligned}
$$

Also, the first order partials of the dilated coefficients are uniformly bounded. 
Let $\widetilde{P}^{k}(x, D)$ denote the operator $\widetilde{P}(x, D)$ with rough coefficients $\tilde{\rho}, \tilde{a}_{i j}$ replaced by smooth coefficients $\tilde{\rho}^{k}, \tilde{a}_{i j}^{k}$. By (5.2), we can now apply the results of Sections 3 and 4 to equations involving the operator $\widetilde{P}^{k}\left(2^{-k \sigma} x, D\right)$. Set $v_{k}(t, x)=$ $\left(\psi \Gamma_{k} u\right)\left(2^{-k \sigma}(t, x)\right)$ so that $\widehat{v_{k}}\left(\tau, x_{1}, \xi^{\prime}\right)$ is localized to frequencies $\approx 2^{k(1-\sigma)}$. Since $\alpha>0$, we have that $\varsigma<1-\sigma$ so Corollary 4.5 now allows us to conclude that

$$
\begin{array}{r}
\left\|v_{k}\right\|_{L_{t}^{p} L_{x}^{q}\left(\mathbb{R} \times 2^{k \sigma} I_{m}^{k} \times \mathbb{R}^{n-1}\right)} \lesssim 2^{k(1-\sigma) s}\left(\left\|v_{k}\right\|_{L_{x_{1}}^{\infty}\left(2^{k \sigma} I_{m}^{k} ; \dot{H}_{t, x^{\prime}}^{1}\right)}+\left\|\partial_{1} v_{k}\right\|_{L_{x_{1}}^{\infty}\left(2^{k \sigma} I_{m}^{k} ; L_{t, x^{\prime}}^{2}\right)}\right. \\
\left.+\left\|\widetilde{P}^{k}\left(2^{-k \sigma} x, D\right) v_{k}\right\|_{L_{x_{1}}^{1}\left(2^{k \sigma} I_{m}^{k} ; L_{t, x^{\prime}}^{2}\right)}\right)
\end{array}
$$

where $2^{k \sigma} I_{m}^{k}$ denotes the set $\left\{2^{k \sigma} x_{1}: x_{1} \in I_{m}^{k}\right\}$ and $\dot{H}^{1}$ denotes the homogeneous $L^{2}$ Sobolev space of order 1 . We now rescale the estimate to get

$$
\begin{aligned}
\left\|\chi_{m} \psi \Gamma_{k} u\right\|_{L_{t}^{p} L_{x}^{q} \lesssim 2^{k s}\left(2^{k} \|\right.} & \psi \Gamma_{k} u \|_{L_{x_{1}}^{\infty} L_{t, x^{\prime}}^{2}} \\
& \left.+\left\|\partial_{1} \psi \Gamma_{k} u\right\|_{L_{x_{1}}^{\infty} L_{t, x^{\prime}}^{2}}^{2}+\left\|\chi_{m} \widetilde{P}_{k}\left(\psi \Gamma_{k} u\right)\right\|_{L_{x_{1}}^{1} L_{t, x^{\prime}}^{2}}\right) .
\end{aligned}
$$

By writing out the expression for $\widetilde{P}-\widetilde{P}^{k}$ it is easy to see that the operator involves differentiation in $x_{1}$ at most one time. We can thus use (5.1) to obtain the estimate

$$
\left\|\chi_{m}\left(\widetilde{P}^{k}-\widetilde{P}\right)\left(\psi \Gamma_{k} u\right)\right\|_{L_{x_{1}}^{1} L_{t, x^{\prime}}^{2}} \leq C\left(2^{k}\left\|\psi \Gamma_{k} u\right\|_{L_{x_{1}}^{\infty} L_{t, x^{\prime}}^{2}}+\left\|\partial_{1} \psi \Gamma_{k} u\right\|_{L_{x_{1}}^{\infty} L_{t, x^{\prime}}^{2}}\right)
$$

This now yields the inequality (5.3) except with $\widetilde{P}^{k}$ replaced by $\widetilde{P}$. Taking the sum over $m$ in this inequality as it ranges over all subintervals $\left\{I_{m}^{k}\right\}$ now yields the following estimate when $p \leq q$

$$
\begin{aligned}
& \left\|\psi \Gamma_{k} u\right\|_{L_{t}^{p} L_{x}^{q}} \leq\left(\int\left(\sum_{m} \int\left|\chi_{m}^{k} \psi \Gamma_{k} u\right|^{q} d x\right)^{\frac{p}{q}} d t\right)^{\frac{1}{p}} \leq\left(\sum_{m}\left\|\chi_{m}^{k} \psi \Gamma_{k} u\right\|_{L_{t}^{p} L_{x}^{q}}^{p}\right)^{\frac{1}{p}} \\
& \text { (5.4) } \leq C 2^{k\left(s+\frac{\sigma}{p}\right)}\left(2^{k}\left\|\psi \Gamma_{k} u\right\|_{L_{x_{1}}^{\infty} L_{t, x^{\prime}}^{2}}+\left\|\partial_{1} \psi \Gamma_{k} u\right\|_{L_{x_{1}}^{\infty} L_{t, x^{\prime}}^{2}}+\left\|P\left(\psi \Gamma_{k} u\right)\right\|_{L_{x_{1}}^{1} L_{t, x^{\prime}}^{2}}\right)
\end{aligned}
$$

To obtain a suitable estimate when $p>q$, we first observe that this implies that $n=3, s=0$ and $p=\frac{2 q}{q-6}$ with $q<8 . H^{1}\left(\mathbb{R}^{3}\right) \hookrightarrow L^{6}\left(\mathbb{R}^{3}\right)$ by Sobolev embedding and hence

$$
\left\|\psi \Gamma_{k} u\right\|_{L_{t}^{\infty} L_{x}^{6}} \leq C\left\|\Gamma_{k} u\right\|_{L_{t}^{\infty} H_{x}^{1}}
$$

We can now use analytic interpolation on this estimate and (5.4) with $s=0$, $p=q=8$ to get that

$$
\begin{gathered}
\left\|\psi \Gamma_{k} u\right\|_{L_{t}^{p} L_{x}^{q} \lesssim 2^{k\left(s+\frac{\sigma}{p}\right)}}\left(\left\|\Gamma_{k} u\right\|_{L_{t}^{\infty} H_{x}^{1}}+2^{k}\left\|\psi \Gamma_{k} u\right\|_{L_{x_{1}}^{\infty} L_{t, x^{\prime}}^{2}}+\left\|\partial_{1} \psi \Gamma_{k} u\right\|_{L_{x_{1}}^{\infty} L_{t, x^{\prime}}^{2}}\right. \\
\left.+\left\|P\left(\psi \Gamma_{k} u\right)\right\|_{L_{x_{1}}^{1} L_{t, x^{\prime}}^{2}}\right)
\end{gathered}
$$

Applying Theorem 2.1, Theorem 1.2 is now shown.

\section{REFERENCES}

[1] Bahouri, H.; Chemin, J.-Y. Equations d'ondes quasilineares et effet dispersif. Int. Math. Res. Not. 1999, 21, 1141-1178.

[2] Bahouri, H.; Chemin, J.-Y. Equations d'ondes quasilineares et estimations de Strichartz. Amer. J. Math. 1999, 121 (6), 1337-1377.

[3] Genibre, J.; Velo, G. Generalized Strichartz inequalities for the wave equation. J. Funct. Anal. 1995, 133 (1), 50-68. 
[4] Keel, M.; Tao, T. Endpoint Strichartz Estimates. Amer. J. Math 1998, 120, 955-980.

[5] Lindblad, H.; Sogge, C. D. On existence and scattering with minimal regularity for semilinear wave equations. J. Funct. Anal. 1995, 130 (2), 357-426.

[6] Smith, H. A Parametrix Construction for Wave Equations with $C^{1,1}$ Coefficients. Ann. Inst. Fourier (Grenoble) 1998, 48 (3), 797-835.

[7] Smith, H.; Sogge, C. On Strichartz and Eigenfunction estimates for low regularity metrics. Math. Res. Lett. 19941 (6), 729-737.

[8] Stein, E. M. Harmonic Analysis: Real Variable Methods, Orthogonality, and Oscillatory Integrals; Princeton University Press: Princeton, N.J., 1993.

[9] Stein, E. M. Singular Integrals and Differentiability Properties of Functions; Princeton University Press: Princeton, N.J., 1970.

[10] Strichartz, R. A priori estimates for the wave equation and some applications. J. Funct. Analysis 1970, 5, 218-235.

[11] Strichartz, R. Restriction of Fourier Transform to quadratic surfaces and decay of solutions to the wave equation. Duke Math J. 1977, 44 (3), 705-714.

[12] Tataru, D. Strichartz estimates for second order hyperbolic operators with nonsmooth coefficients and the nonlinear wave equation. Amer. J. Math 2000, 122 (2), 349-376.

[13] Tataru, D. Strichartz estimates for second order hyperbolic operators with nonsmooth coefficients II. Amer. J. Math 2001, 123 (3), 385-423.

[14] Tataru, D. Strichartz estimates for second order hyperbolic operators with nonsmooth coefficients III. J. Amer. Math. Soc. 2002, 15 (2), 419-442.

[15] Taylor, M.E. Pseudodifferential Operators and Nonlinear PDE; Birkhhäuser: Boston, 1991.

Department of Mathematics, University of Washington, Seattle, WA 98195

E-mail address: blair@math.washington.edu 